\title{
Thermal- Elastic Waves of Microstretch Semiconductor Medium with Rotation Field during Photothermal Excitation
}

khaled lotfy ( $\sim$ khlotfy_1@yahoo.com )

Zagazig University Faculty of Science https://orcid.org/0000-0001-9383-1361

\section{A. A. El-Bary}

Arab Academy for Science Technology and Maritime Transport

\section{Research Article}

Keywords: Photo-thermoelasticity theory, Semiconductor, Rotation, Microstretch, Microinertia, Harmonic wave

Posted Date: May 25th, 2021

DOI: https://doi.org/10.21203/rs.3.rs-394799/v1

License: (c) (i) This work is licensed under a Creative Commons Attribution 4.0 International License.

Read Full License 


\title{
Thermal- Elastic Waves of Microstretch Semiconductor Medium with Rotation Field during Photothermal Excitation
}

\author{
Kh. Lotfy ${ }^{1,3}$ and A. A. El-Bary ${ }^{2,4}$ \\ ${ }^{1}$ Department of Mathematics, Faculty of Science, Zagazig University, P.O. Box44519, Zagazig, Egypt \\ ${ }^{2}$ Arab Academy for Science, Technology and Maritime Transport, P.O. Box 1029, Alexandria, Egypt. \\ ${ }^{3}$ Department of Mathematics, College of Science, Taibah University, Madinah., Saudi Arabia. \\ ${ }^{4}$ National Committee for Mathematics, Academy of Scientific Research and Technology, Egypt.
}

khlotfy_1@yahoo.com, aaelbary@aast.edu

\begin{abstract}
A mathematical novel model for elastic semiconductor medium with microstretch properties is investigated. The generalized model is studied in the context of photo-thermoelasticity theory when the semiconductor medium is excited. The governing equations describe the coupled between the propagation of the elastic-thermal-plasma waves when the thermo-microstretch elastic semiconductor material is studied during a rotation field. The linear medium has an isotropic properties. The photothermal transport processes occurs during a two dimension (2D) elastic and electronic deformation when the microinertia of microelement is taken into account. The harmonic wave method can be used to obtain the general solutions for the basic physical variables. The complete analytical solutions of the considered variables are obtained when some mechanical-thermal and plasma conditions are applied on the boundary of the semiconductor medium. The numerical simulations of silicon ( $\mathrm{Si}$ ) and Germanium (Ge) media are constructed graphically with many comparisons according to a new parameters with thermal memories and rotation parameter.
\end{abstract}

Keywords: Photo-thermoelasticity theory; Semiconductor; Rotation; Microstretch; Microinertia; Harmonic wave. 


\section{Nomenclature}

$\lambda, \mu \quad$ Lame's elastic parameters.

$\delta_{n} \quad$ The deformation potential difference.

$T \quad$ The thermodynamic heat.

$T_{0} \quad$ Temperature in its natural state (reference) which it satisfys $\left|\frac{T-T_{0}}{T_{0}}\right|<1$. $\hat{\gamma}=(3 \lambda+2 \mu+k) \alpha_{t_{1}} \quad$ The volume thermal expansion.

$\sigma_{i j} \quad$ The stress coefficient tensor.

$\rho \quad$ The density of the sample.

$\alpha_{t_{1}}, \alpha_{t_{2}} \quad$ Coefficients of linear thermal expansion.

e Cubical dilatation.

$C_{e} \quad$ Specific heat of the material at constant strain.

$k \quad$ The thermal conductivity.

$D_{E} \quad$ The carrier diffusion coefficient.

$\tau \quad$ The carrier lifetime.

$E_{g} \quad$ The energy gap.

$e_{i j} \quad$ Components of strain tensor.

$\Pi, \Psi \quad$ Two scalar functions.

$j_{0} \quad$ The microinertia of microelement.

$m_{i j} \quad$ Couple stress tensor.

$\alpha_{0}, \lambda_{0}, \lambda_{1} \quad$ Microstretch elastic constants.

$\tau_{0}, v_{0} \quad$ Thermal relaxation times.

$\varphi \quad$ Rotation inertia vector.

$\varphi^{*} \quad$ The scalar microstretch.

$\lambda_{i} \quad$ The microstress (first moment) tensor 


\section{Introduction}

Semiconductor materials have great importance and uses in modern industry. As these materials do not conduct electricity under normal conditions and have resistance to the internal movement of electrons. But when the semiconductor medium exposed to some external influences such as a change in temperature and resistance decreases, its internal properties also change. When the change in body temperature is not only the result of exposure of the medium to external and internal heat sources, but also deformations of the process itself during the microinertia process of the microelements. These changes occur during two processes, the thermo-elastic and the electron deformation (TD and ED respectively). During these processes may be prescribed the mechanical, thermal, plasma waves distribution. Plasma waves appear as a result of excited electrons on the surface of the semiconducting material which move freely in what is known as carrier density. On the other hand, thermal and mechanical waves occur as a result of the movement and collisions of internal particles of the elastic semiconducting material. In this case, the thermal and mechanical influences have a relation with stresses and strains that occur in elastic semiconductor body. In any case, as a result of these overlaps, the linear micropolar photo-thermoelasticity theory can be taken into consideration. In this case, the body microstructure impact is very important and this impact gives a new results of wave distributions can't be exist in classical photo-thermoelasticity theory.

The microstructure of body is studied during the thermal-elastic interaction processes of isotropic homogeneous elastic body in the context of the generalized thermoelasticity theory [1]. The microstructure impacts on the wave propagations are developed by Eringen and Şuhubi [2]. The new theory with micropolar influence is constructed in the context of the generalized thermoelasticity theory by Eringen [3]. Recently, the new theory is called microstretch thermoelasticity theory 
which it appears as a special case of theory of micromorphic. After that many authors developed the generalized microstretch thermoelasticity theory with micropolar when they studied the reflection and refraction waves with two temperatures theory during a liquid adjacent to an elastic thermo-microstretch medium [4-6]. The linear generalized thermo-microstretch of elastic media studied the heat conduction as a thermal waves with a finite speed [7]. Lotfy and Othman et al. [8,9] used the effect of external gravitational field, magnetic field and hydrostatic initial stress with the thermal memories influences during the studying of the generalized -thermo-microstretch theory. Many applications of thermomicrostretch theory during a hydromechanics viscoelastic porous media are used with the thermal radiation effect $[10,11]$. On the other hand, Ezzat et al. $[12,13]$ used the state space approach to study the viscoelastic fluid porous medium flow under the effect of magnetic field and perfectly conducting for boundary-layer over a stretching wall. Carrera and Valvano $[14,15]$ studied a variable kinematic for shell and plate formulation for when used thermal stresses analysis of laminated structures. On the other hand, Cinefra et al. [16, 17] investigated the thermal stress analysis and Heat conduction of laminated composites by a variable kinematic shell element.

When a laser beams or light fall on an intracavity spherical semiconductor medium, the photothermal phenomenon is appeared [8]. The spectroscopy of photoacoustic with a sensitive analysis is used obtain the real measuring of thermal, mechanical and plasma waves of semiconducting material to [9]. Many applications in mechanical and electrical engineering used ultrasensitive laser spectroscopy with photothermal transport process [20-22]. Recently, the photothermal effect of semiconductor elastic materials during 2D thermal and electronic deformation is studied [23]. The generalized thermoelastic vibrations during the electronic deformation mechanism are studied in the context of the 
optically excited to discuss the spectroscopy of photoacoustic analysis [24, 25]. Lotfy et al. [26-32] developed many physical problems in a new photothermoelasticity theory when they studied a different applications in modern physics. A dual phase-lags models are used to study photothermal excitation during the interaction processes of a semiconductor materials [33, 34]. After that, the memory responses of the photo-thermoelasticity theory when the physical properties of the semiconductor elastic medium depend on the change of the temperature with many external fields are studied [35-39]. Ezzat [40, 41] investigated The hyperbolic and fractional thermal-plasma-elastic wave propagations is studied in a non-metallic semiconductor of organic medium.

In all the above investigations the optical properties and the inner structure of the semiconductor material are not taken into account. But in this work the governing equations are studied under the effect of rotation when the interaction between the microstretch (inner-structure) theory and the generalized photothermoelasticiy occur. In this case, the microinertia of microelement are taken during 2D (in the space $(x, z)$ ) thermo-elastic and electronic deformation. The main equations describe the physical variables distributions in the generalized photo-thermo-microstretch semiconductor elastic medium with various thermal memories. The harmonic wave with normal mode technique is used when a different mechanical-thermal-plasma conditions are applied at the free surface. Some algebraic techniques with numerical calculations are used to obtain the complete main solutions of the physical quantities. The obtained results are shown with some comparisons graphically and discussed.

\section{Mathematical model and main equations}

Many authors over the years constructed many models in the theory of the generalized thermoelasticity [38-40]. During the generalized photo-thermo- 
microstretch semiconductor medium which is taken in a Cartesian coordinates. The photothermal with optical mechanism (carrier charges (density) are generated and the plasma wave propagation appear ) is generated at the free surface is due to the thermal effects. The interaction processes between the plasma-thermal-elastic waves during the transmission microstretch excitation are occurred. In the case of uniform rotation field with angular velocity $\underline{\Omega}=\Omega \underline{n}$ of a semiconductor medium around the axial y (where $\underline{n}$ is a unit vector in the direction of y-axis) (see the geometry of the problem). The governing equations which describe a 2D photothermo-microstretch theory under the effect of rotation can be obtained as follow:

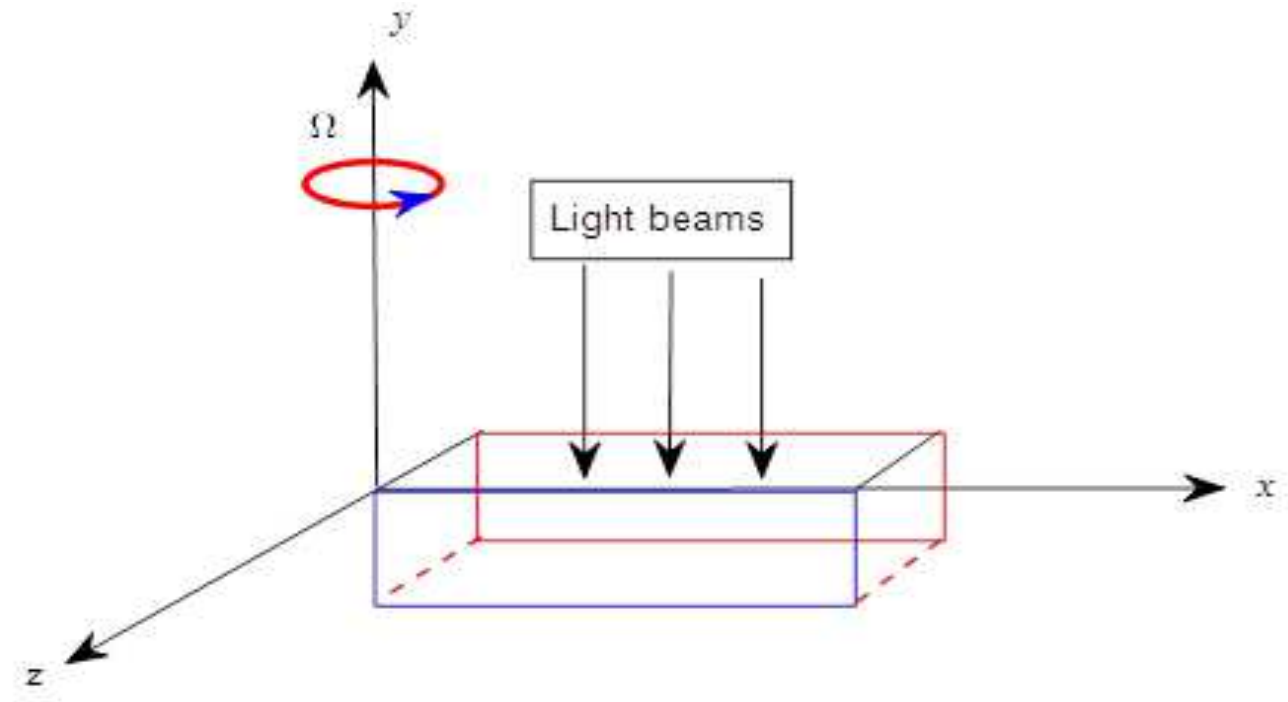

Geometry of the problem

(I) The interaction between thermal distribution and carrier density (plasma wave) in the context of the microstraetch photo-thermal excitation process can be given as [23]:

$$
\frac{\partial N}{\partial t}=D_{E} \nabla^{2} N-\frac{N}{\tau}+\kappa T
$$

(II) The equations of motion during the microstraetch photo-thermal transport process under the effect of a uniform rotation vector when the 
microinertia process of the microelements is taken into account can be written as follows [42]:

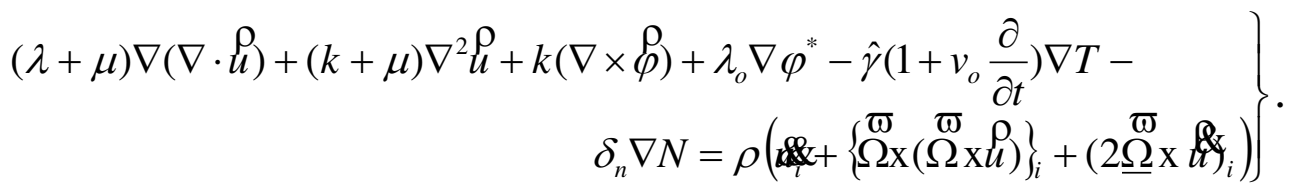

$$
\begin{aligned}
& (\alpha+\beta+\gamma) \nabla(\nabla \cdot \rho)-\gamma \nabla \times(\nabla \times \rho)+k(\nabla \times \ell)-2 k \rho=j \rho \phi . \\
& \alpha_{o} \nabla^{2} \varphi^{*}-\frac{1}{3} \lambda_{1} \varphi^{*}-\frac{1}{3} \lambda_{o}(\nabla \cdot \hat{u})+\frac{1}{3} \hat{\gamma}_{1}\left(1+v_{o} \frac{\partial}{\partial t}\right) T=\frac{3}{2} j \rho .
\end{aligned}
$$

(III) The heat conduction equation which describe the coupling between the photo-thermal-elastic-microstretch processes of semiconductor medium can be given as [19]:

$$
K \nabla^{2} T-\rho C_{E}\left(n_{1}+\tau_{o} \frac{\partial}{\partial t}\right) T^{\&} \hat{\gamma} T_{o}\left(n_{1}+n_{o} \tau \frac{\partial}{\partial t}\right) \&+\frac{E_{g}}{\tau K} N=\hat{\gamma}_{1} T_{o} \text { \& }
$$

(IV) The constitutive relations for the elastic microstretch semiconductor material during the photo-thermoelasticity theory which can be given as:

$$
\begin{gathered}
m_{i l}=a \varphi_{r},{ }_{r} \delta_{i l}+\beta \varphi_{i, l}+\gamma \varphi_{l, i}, \\
\lambda_{i}=a_{o} \varphi^{*} .
\end{gathered}
$$

(V) The tensor form of the constitutive equations for generalized photothermo-microstretch theory can be obtained as [43]:

$$
\sigma_{i I}=\left(\lambda_{o} \varphi^{*}+\lambda u_{r}, r\right) \delta_{i I}+(k+\mu) u_{I i}-k \varepsilon_{i I} \varphi_{r}-\hat{\gamma}\left(1+v_{o} \frac{\partial}{\partial t}\right) T \delta_{i I}-\left((3 \lambda+2 \mu+k) d_{n} N\right) \delta_{i I} .
$$

In the context of a $2 \mathrm{D}$ deformation, the displacement vector $\tilde{u}$, the scalar microstretch function and rotation vector can be analyzed in $x z$-plane as follows:

$$
\left.\begin{array}{c}
u=(u, 0, w) ; u=u(x, z, t), w=w(x, z, t), \\
\rho=\left(0, \varphi_{2}, 0\right) ; \varphi_{2}=\varphi_{2}(x, z, t), \varphi^{*}=\varphi^{*}(x, z, t)
\end{array}\right\} .
$$

The 2D strain relation in terms of the displacement components can be given as:

$$
e=\frac{\partial u}{\partial x}+\frac{\partial w}{\partial z}
$$


Where in general case the parameter $\kappa=\frac{\partial n_{0}}{\partial T}$ is the coupling thermal activation and $\hat{\gamma}_{1}=(3 \lambda+2 \mu+k) \alpha_{t_{2}}$ represents an parameter that it depends on the thermalmicrostretch properties, $\alpha_{t_{2}}$ expresses a coefficient of the linear thermalmicrostretch expansions.

In $2 \mathrm{D}$ deformations, the governing equations (2)-(5) can be rewritten as:

$$
\begin{aligned}
& (\lambda+\mu)\left(\frac{\partial^{2} u}{\partial x^{2}}+\frac{\partial^{2} w}{\partial x \partial z}\right)+(k+\mu)\left(\frac{\partial^{2} u}{\partial x^{2}}+\frac{\partial^{2} u}{\partial z^{2}}\right)-k \frac{\partial \varphi_{2}}{\partial z}+ \\
& \left.\lambda_{o} \frac{\partial \varphi^{*}}{\partial x}-\hat{\gamma}\left(1+v_{o} \frac{\partial}{\partial t}\right) \frac{\partial T}{\partial x}-\delta_{n} \frac{\partial N}{\partial x}=\rho\left(\frac{\partial^{2} u}{\partial t^{2}}-\Omega^{2} u+2 \Omega \frac{\partial w}{\partial t}\right)\right\}, \\
& (\lambda+\mu)\left(\frac{\partial^{2} u}{\partial x \partial z}+\frac{\partial^{2} w}{\partial z^{2}}\right)+(k+\mu)\left(\frac{\partial^{2} w}{\partial x^{2}}+\frac{\partial^{2} w}{\partial z^{2}}\right)-k \frac{\partial \varphi_{2}}{\partial x}+ \\
& \left.\lambda_{o} \frac{\partial \varphi^{*}}{\partial z}-\hat{\gamma}\left(1+v_{o} \frac{\partial}{\partial t}\right) \frac{\partial T}{\partial z}-\delta_{n} \frac{\partial N}{\partial z}=\rho\left(\frac{\partial^{2} w}{\partial t^{2}}-\Omega^{2} w-2 \Omega \frac{\partial u}{\partial t}\right)\right\}, \\
& \gamma\left[\frac{\partial^{2} \varphi_{2}}{\partial x^{2}}+\frac{\partial^{2} \varphi_{2}}{\partial z^{2}}\right]+k\left(\frac{\partial u}{\partial z}-\frac{\partial w}{\partial x}\right)-2 k \varphi_{2}=j \rho \frac{\partial^{2} \varphi_{2}}{\partial t^{2}}, \\
& \alpha_{o}\left[\frac{\partial^{2} \varphi^{*}}{\partial x^{2}}+\frac{\partial^{2} \varphi^{*}}{\partial z^{2}}\right]-\frac{1}{3} \lambda_{1} \varphi^{*}-\frac{1}{3} \lambda_{o}\left(\frac{\partial u}{\partial x}+\frac{\partial w}{\partial z}\right)+\frac{1}{3} \hat{\gamma}_{1}\left(1+v_{o} \frac{\partial}{\partial t}\right) T=\frac{3}{2} j \rho \frac{\partial^{2} \varphi^{*}}{\partial t^{2}}, \\
& K\left[\frac{\partial^{2} T}{\partial x^{2}}+\frac{\partial^{2} T}{\partial z^{2}}\right]-\rho C_{E}\left(n_{1}+\tau_{o} \frac{\partial}{\partial t}\right) \frac{\partial T}{\partial t}-\hat{\gamma} T_{o}\left(n_{1}+n_{o} \tau_{o} \frac{\partial}{\partial t}\right) \frac{\partial e}{\partial t}+\frac{E_{g}}{\tau K} N=\hat{\gamma}_{1} T_{o} \frac{\partial \varphi^{*}}{\partial t} \text {. }
\end{aligned}
$$

According to the photo-thermal-microstretch theories, the thermal relaxation times and the parameters $n_{o}, n_{1}$ (are constants) can be chosen arbitrary subjected to classical coupled model (CD), Lord and Şhulman (LS) theory and Green and Lindsay (GL) model.

The following dimensionless form can be introduced for more simplification: 


$$
\begin{aligned}
& \bar{N}=\frac{\delta_{n}}{2 \mu+\lambda} N, \quad \bar{x}_{i}=\frac{\omega^{*}}{C_{2}} x_{i}, \quad \bar{u}_{i}=\frac{\rho C_{2} \omega^{*}}{T_{o} \hat{\gamma}} u_{i}, \quad \bar{t}=\omega^{*} t, \quad \bar{\tau}_{o}=\omega^{*} \tau_{o}, \\
& \bar{v}_{o}=\omega^{*} v_{o}, \quad \bar{T}=\frac{T}{T_{o}}, \quad \bar{\sigma}_{i j}=\frac{\sigma_{i j}}{T_{o} \hat{\gamma}}, \quad \Omega^{\prime}=\frac{\Omega}{\omega^{*}}, \bar{m}_{i j}=\frac{\omega^{*}}{C_{2} T_{o} \hat{\gamma}} m_{i j}, \quad \bar{\varphi}_{2}=\frac{\rho C_{2}^{2}}{T_{o} \hat{\gamma}} \varphi_{2}, \\
& \bar{\lambda}_{3}=\frac{\omega^{*}}{C_{2} T_{o} \hat{\gamma}} \lambda_{3}, \quad \bar{\varphi}^{*}=\frac{\rho C_{2}^{2}}{T_{o} \hat{\gamma}_{1}} \varphi^{*}, \quad \omega^{*}=\frac{\rho C_{E} C_{2}^{2}}{K}, \quad C_{2}^{2}=\frac{\mu}{\rho} .
\end{aligned} .
$$

The non-dimensional equation (16) can be used to simplify the governing equations (with dropping the prim), yields:

$$
\left.\begin{array}{c}
\left(\nabla^{2}-\varepsilon_{3}-\varepsilon_{2} \frac{\partial}{\partial t}\right) N+\varepsilon_{4} T=0, \\
\frac{\partial^{2} u}{\partial t^{2}}-\Omega^{2} u+2 \Omega \frac{\partial w}{\partial t}=\frac{(\lambda+\mu)}{\rho C_{2}^{2}} \frac{\partial e}{\partial x}+\frac{(k+\mu)}{\rho C_{2}^{2}} \nabla^{2} u-\frac{k}{\rho C_{2}^{2}} \frac{\partial \varphi_{2}}{\partial z}+ \\
\frac{\lambda_{o}}{\rho C_{2}^{2}} \frac{\partial \varphi^{*}}{\partial x}-\left(1+v_{o} \frac{\partial}{\partial t}\right) \frac{\partial T}{\partial x}-\frac{(\lambda+2 \mu)}{\gamma T_{o}} \frac{\partial N}{\partial x}
\end{array}\right\},
$$

The potential scalar functions as $\Pi(x, z, t)$ and vector space-time function $\Psi(x, z, t)=(0, \psi, 0)$, can be introduced to simplify the above field equations. The dimensionless of the displacement vector can be presented in terms of the potential scalar and vector functions according to Helmholtz's theory as follows: 


$$
\tilde{u}=\operatorname{grad} \Pi+\operatorname{curl} \Psi, u=\frac{\partial \Pi}{\partial x}-\frac{\partial \psi}{\partial z}, w=\frac{\partial \Pi}{\partial \mathrm{z}}+\frac{\partial \psi}{\partial x} .
$$

Therefore, equation (23) can be used of the main equations (18)-(22) for more simplification, in this case equations (17)-(22) can be simplified as:

$$
\begin{gathered}
\left(\nabla^{2}+a_{o} \Omega^{2}-a_{12} \frac{\partial^{2}}{\partial t^{2}} \Pi+a_{o}\left(1+v_{o} \frac{\partial}{\partial t}\right) T+2 \Omega a_{o} \frac{\partial \psi}{\partial t}+a_{1} \varphi^{*}-a_{4} N=0,\right. \\
\left(\nabla^{2}+a_{2} \Omega^{2}-a_{2} \frac{\partial^{2}}{\partial t^{2}}\right) \psi-a_{13} \frac{\partial \Pi}{\partial t}-a_{3} \varphi_{2}=0, \\
\left(\nabla^{2}-2 a_{5}-a_{6} \frac{\partial^{2}}{\partial t^{2}}\right) \varphi_{2}-a_{7} \nabla^{2} \psi=0, \\
\left(a_{8} \nabla^{2}-a_{10}-\frac{\partial^{2}}{\partial t^{2}}\right) \varphi^{*}-a_{11} \nabla^{2} \Pi+a_{9}\left(1+v_{o} \frac{\partial}{\partial t}\right) T=0, \\
{\left[\nabla^{2}-\left(n_{1} \frac{\partial}{\partial t}+\tau_{o} \frac{\partial^{2}}{\partial t^{2}}\right)\right] T-\varepsilon\left(n_{1} \frac{\partial}{\partial t}+n_{o} \tau_{o} \frac{\partial^{2}}{\partial t^{2}}\right) \nabla^{2} \Pi+\varepsilon_{5} N-\varepsilon_{1} \frac{\partial \varphi^{*}}{\partial t}=0 .}
\end{gathered}
$$

Here,

$$
\left.\begin{array}{l}
a_{o}=\frac{\rho c_{2}^{2}}{2 \mu+k+\lambda}, \quad a_{1}=\frac{\lambda_{o}}{2 \mu+k+\lambda}, \quad a_{2}=\frac{\rho c_{2}^{2}}{\mu+k}, \quad a_{3}=\frac{k}{\mu+k}, \\
a_{4}=\frac{\rho c_{2}^{2}(2 \mu+\lambda)}{\hat{\gamma} T_{o}(2 \mu+k+\lambda)}, a_{5}=\frac{k c_{2}^{2}}{\gamma \omega^{*}}, \quad a_{6}=\frac{j \rho c_{2}^{2}}{\gamma}, \quad a_{7}=\frac{k C_{2}^{2}}{\gamma \omega^{* 2}}, \quad a_{8}=\frac{C_{3}^{2}}{C_{2}^{2}}, \\
a_{9}=\frac{2 \hat{\gamma}_{1} C_{2}^{2}}{9 \hat{\gamma} j \omega^{* 2}}, \quad a_{10}=\frac{C_{4}^{2}}{\omega^{* 2}}, \quad a_{11}=\frac{C_{5}^{2}}{\omega^{* 2}}, \quad \varepsilon=\frac{\hat{\gamma}_{o}}{K \rho \omega^{*}}, \quad \varepsilon_{1}=\frac{\hat{\gamma} T_{o}}{K \rho \omega^{*}}, \\
\varepsilon_{2}=\frac{C_{2}^{2}}{D_{E} \omega^{*}}, \quad \varepsilon_{3}=\frac{C_{2}^{2}}{\tau D_{E} \omega^{* 2}}, \quad \varepsilon_{4}=\frac{\kappa T_{o} \delta_{n} C_{2}^{2}}{D_{E}(2 \mu+\lambda) \omega^{* 2}}, \varepsilon_{5}=\frac{E_{g}(2 \mu+\lambda) C_{2}^{2}}{\tau K^{2} T_{o} \omega^{* 2} \delta_{n}}, \\
C_{3}^{2}=\frac{2 \alpha_{o}}{3 \rho j}, \quad C_{4}^{2}=\frac{2 \lambda_{1}}{9 \rho j}, \quad C_{5}^{2}=\frac{2 \lambda_{o}}{9 \rho j}, \quad C_{6}^{2}=\frac{2 \hat{\gamma}_{1}}{9 \rho j}, a_{12}=2 a_{o} \Omega, a_{13}=2 a_{2} \Omega .
\end{array}\right\} .
$$

\section{Harmonic wave analysis}

To evaluate the analytical solutions in $2 \mathrm{D}$ deformation of the main physical quantities which propagated in the plane wave, in this case the physical fields can be constructed by the harmonic waves (normal mode technique) method as [4042]: 


$$
\left.\begin{array}{l}
\left(\varphi, \psi, \varphi^{*}, \varphi_{2}, \sigma_{i I}, T, m_{i I}, N\right)(x, z, t)= \\
\left(\bar{\varphi}(x), \bar{\psi}(x), \bar{\varphi}^{*}(x), \bar{\varphi}_{2}(x), \bar{\sigma}_{i I}(x), \bar{T}(x), \bar{m}_{i I}(x), \bar{N}(x)\right) \exp (\omega t+i b z)
\end{array}\right\} .
$$

However, the quantities $\bar{\varphi}, \bar{\psi}, \bar{\varphi}^{*}, \bar{\varphi}_{2}, \bar{\sigma}_{i l}, \bar{m}_{i l}, \bar{T}, \bar{N}$ represent the amplitude values of the main field quantities, $i=\sqrt{-1}, \omega$ is frequency of complex time and $b$ expresses the wave number in the direction of $z$-axis. Using equation (30) to convert the partial differential equations (17) and (24)-(28) to ordinary for equations as follow:

$$
\begin{gathered}
\left(D^{2}-\alpha_{1}\right) \bar{N}+\varepsilon_{4} \bar{T}=0, \\
\left(D^{2}-A_{1}\right) \bar{\Pi}+A_{2} \bar{T}+A_{9} \bar{\psi}+a_{1} \bar{\varphi}^{*}-a_{4} \bar{N}=0, \\
\left(D^{2}-A_{3}\right) \bar{\psi}-a_{3} \bar{\varphi}_{2}-a_{14} \bar{\Pi}=0, \\
\left(D^{2}-A_{4}\right) \bar{\varphi}_{2}-a_{7}\left(D^{2}-b^{2}\right) \bar{\psi}=0, \\
\left(a_{8} D^{2}-A_{5}\right) \bar{\varphi}^{*}-a_{11}\left(D^{2}-b^{2}\right) \bar{\Pi}+A_{8} \bar{T}=0, \\
\left(D^{2}-A_{6}\right) \bar{T}-A_{7}\left(D^{2}-b^{2}\right) \bar{\Pi}+\varepsilon_{5} \bar{N}-\varepsilon_{1} \omega \bar{\varphi}^{*}=0 .
\end{gathered}
$$

Where,

$$
\begin{aligned}
& \alpha_{1}=b^{2}+\varepsilon_{3}+\varepsilon_{2} \omega, \quad A_{1}=b^{2}+a_{o} \Omega^{2}+a_{o} \omega^{2}, \quad A_{2}=a_{o}\left(1+v_{o} \omega\right), \quad A_{3}=b^{2}+a_{2} \Omega^{2}+a_{2} \omega^{2}, \\
& D=\frac{d}{d \mathrm{x}}, \quad A_{4}=b^{2}+2 a_{5}+a_{6} \omega^{2}, \quad A_{5}=a_{8} b^{2}+a_{10}+\omega^{2}, \quad A_{6}=b^{2}+n_{1} \omega+\tau_{o} \omega^{2}, \\
& \nabla^{2}=D^{2}, \quad A_{7}=\varepsilon\left(n_{1} \omega+n_{o} \tau_{o} \omega^{2}\right), \quad A_{8}=a_{9}\left(1+v_{o} \omega\right), A_{9}=2 \Omega \omega a_{o}, a_{14}=a_{13} \omega .
\end{aligned}
$$

Solving the above system of equations (31)-(36) in terms $\bar{\Pi}(x)$, in this case the following ordinary differential equation (ODE) can be obtain as (which they satisfied by all quantities) form:

$$
\left(D^{12}-\Theta_{1} D^{10}+\Theta_{2} D^{8}-\Theta_{3} D^{6}+\Theta_{4} D^{4}-\Theta_{5} D^{2}+\Theta_{6}\right)\left[\frac{\hbar}{\Pi}, \bar{T}, \bar{\varphi}^{*}, \bar{\varphi}_{2}, \bar{\psi}, \bar{N}\right\}(x)=0 .
$$

According to the linearity property, the solutions of the above ODE equation (38) can be written in the following form:

$$
\bar{\Pi}(x)=\sum_{n=1}^{6} \Gamma_{n}(b, \omega) \exp \left(-l_{n} x\right),
$$




$$
\begin{gathered}
\bar{\varphi}^{*}(x)=\sum_{n=1}^{6} \Gamma_{n}^{\prime}(b, \omega) \exp \left(-l_{n} x\right), \\
\bar{T}(x)=\sum_{n=1}^{6} \Gamma_{n}^{\prime \prime}(b, \omega) \exp \left(-l_{n} x\right), \\
\bar{N}(x)=\sum_{n=1}^{6} \Gamma_{n}^{\prime \prime \prime}(b, \omega) \exp \left(-l_{n} x\right), \\
\bar{\psi}(x)=\sum_{n=1}^{6} \Gamma_{n}^{\prime \prime \prime}(b, \omega) \exp \left(-k_{j} x\right), \\
\bar{\varphi}_{2}(x)=\sum_{n=1}^{6} \Gamma_{n}^{\prime \prime \prime \prime}(b, \omega) \exp \left(-k_{j} x\right)
\end{gathered}
$$

Where, $\Gamma_{n}$, the ondepend that parametersunknown sixare $\Gamma_{n}^{\prime \prime \prime \prime \prime}$ and $\Gamma_{n}^{\prime \prime \prime \prime}, \Gamma_{n}^{\prime \prime \prime}, \Gamma_{n}^{\prime \prime}, \Gamma_{n}^{\prime}$ wave number $\mathrm{b}$ and complex time $\omega$. However, the ODE equation (38) can be put in a factorization form as:

$$
\left(D^{2}-k_{1}^{2}\right)\left(D^{2}-k_{2}^{2}\right)\left(D^{2}-k_{3}^{2}\right)\left(D^{2}-k_{4}^{2}\right)\left(D^{2}-k_{5}^{2}\right)\left(D^{2}-k_{6}^{2}\right) \bar{\Pi}(x)=0 \text {. }
$$

On the other hand, the quantities $k_{\mathrm{n}}^{2},(\mathrm{n}=1, \ldots, 6)$ are the basic real roots $\left(\operatorname{Re}\left(k_{n}\right)>0\right)$ of the following characteristic equation:

$$
k^{12}-B_{1} k^{10}+B_{2} k^{8}-B_{3} k^{6}+B_{4} k^{4}-B_{5} k^{2}+B_{6}=0 .
$$

The relations between $\Gamma_{n}$,obtained can be parameters $\Gamma_{n}^{\prime \prime \prime \prime \prime}$ nd a $\Gamma_{n}^{\prime \prime \prime \prime}, \Gamma_{n}^{\prime \prime \prime}, \Gamma_{n}^{\prime \prime}, \Gamma_{n}^{\prime}$ from the basic equations (31)-(36) as follows:

$$
\left.\begin{array}{c}
\bar{\varphi}^{*}(x)=\sum_{n=1}^{6} \Gamma_{n} \mathrm{E}_{1} e^{-k_{n} x}, \bar{T}(x)=\sum_{n=1}^{6} \Gamma_{n} \mathrm{E}_{2} e^{-k_{n} x}, \bar{N}(x)=\sum_{n=1}^{6} \Gamma_{n} \mathrm{E}_{3} e^{-k_{n} x}, \\
\bar{\psi}(x)=\sum_{n=1}^{6} \Gamma_{n} \mathrm{E}_{4} e^{-k_{n} x}, \bar{\varphi}_{2}(x)=\sum_{n=1}^{6} \Gamma_{n} \mathrm{E}_{5} e^{-k_{n} x} .
\end{array}\right\} .
$$

On the other hand, the displacement components in terms of the unknown parameters $\Gamma_{n}$ can be obtained from equations (23), (30) and (47), which can be rewritten in the following form:

$$
\bar{u}(x)=\sum_{n=1}^{6}-\Gamma_{n}\left(k_{n}+i b \mathrm{E}_{4}\right) e^{-k_{n} x}, \bar{w}(x)=\sum_{n=1}^{6}\left(i b-k_{n} \mathrm{E}_{4}\right) \Gamma_{n} e^{-k_{n} x} .
$$


However, the stress-strain relations can be rewritten as:

$$
\bar{\sigma}_{z z}(x)=\sum_{n=1}^{6} s_{n} \Gamma_{n} e^{-k_{n} x}, \quad \bar{\sigma}_{x z}(x)=\sum_{n=1}^{6} r_{n} \Gamma_{n} e^{-k_{n} x} .
$$

The constitutive equations for the microstretch photo-thermoelastic semiconductor medium in dimensionless form can be written as:

$$
\bar{m}_{x y}(x)=\sum_{n=1}^{6} \Gamma_{n} \Lambda_{1} \mathrm{E}_{5} e^{-k_{n} x}, \quad \lambda_{z}=\sum_{n=1}^{6} \Gamma_{n} \Lambda_{2} \mathrm{E}_{1} e^{-k_{n} x} .
$$

Where $\Lambda_{1}=\frac{\gamma \omega^{* 2}}{\rho C_{2}^{4}}$ and $\Lambda_{2}=\frac{\alpha_{0} a_{0} \omega^{* 2}}{\rho C_{2}^{4}}$.

\section{Boundary conditions}

In this section, some conditions are applied at the free surface of the microstretch photo-thermoelastic semiconductor medium (the vertical plan) to obtain the values of the undetermined parameters $\Gamma_{n}$. The mechanical loads with isolated thermally condition and microstretch photo-thermoelastic conditions in the context of a recombination plasma process at the free surface can be chosen as [43]:

$$
\sigma_{z z}=-p=-p^{*} \exp (\omega t+i b z) \quad, \quad \sigma_{x z}=0, \quad m_{x y}=0, \lambda_{z}=0, \quad T=0, \quad \frac{d N}{d x}=-\frac{S_{0} N}{D_{E}} .
$$

Applying the boundary conditions equation (57) with used the harmonic wave technique (30) and the main obtained equations (49)-(50) and (47), in this case the system of six equations when $x=0$ can be obtained:

$$
\sum_{n=1}^{6} s_{n} \Gamma_{n}=-p^{*}, \sum_{n=1}^{6} r_{n} \Gamma_{n}=0, \sum_{n=1}^{6} \Lambda_{1} \mathrm{E}_{5} \Gamma_{n}=0, \sum_{n=1}^{6} \Lambda_{2} \mathrm{E}_{1} \Gamma_{n}=0, \sum_{n=1}^{6} \mathrm{E}_{2} \Gamma_{n}=0, \sum_{n=1}^{6} k_{n} \Gamma_{n}=\frac{S_{0} N}{D_{E}} .
$$

Solving the above system of equations (52) by using the invers of matrix with helping the computer program (Mathematica) the unknown parameters can be obtained.

\section{Validation}

\section{1. The theory of generalized microstretch-thermoelasticity}


When carrier density $N(\stackrel{\omega}{r}, t)$ is neglected (i.e. $N=0)$ in the main equations, the problem is discussed in the generalized microstretch thermoelasticity theory only [22].

\section{2. The generalized photo-thermoelasticity theory}

The generalized photo-thermoelasticity theory is studied only when the microstretch parameters $\alpha_{o}=\lambda_{o}=\lambda_{1}=\varphi^{*}=0$ are ignored. In this case, the governing equations are written in the generalized photo-thermal-elastic for semiconductor medium without stretch [35].

\section{3. Different theories of the microstretch photo-thermoelasticity}

The effect of thermal relaxation times can be obtained during the governing equations of microstretch photo-thermoelasticity processes according to the following relations [44]:

(I) The CD model is obtained when $n_{1}=1, n_{o}=\tau_{o}=v_{o}=0$ [44].

(II) The LS model is obtained when $n_{1}=n_{o}=1, \quad v_{o}=0, \tau_{o}>0,[42]$.

(III) The GL model is observed when $n_{1}=1, \quad n_{o}=0, \quad v_{o} \geq \tau_{o}>0$, [43].

\section{Discussion and numerical results}

To carry out the numerical simulations for this work the semiconductor materials for example silicone $(\mathrm{Si})$ and germanium $(\mathrm{Ge})$ are used with helping the Matlab computer program. The physical (elastic, optical and thermal) constants of $\mathrm{Si}$ and Ge are used to discuss the wave propagation of the main physical fields. The constants of Si material is given in SI unit as [45- 51]:

$$
\begin{aligned}
& \lambda=3.64 \times 10^{10} \mathrm{~N} / \mathrm{m}^{2}, \mu=5.46 \times 10^{10} \mathrm{~N} / \mathrm{m}^{2}, \rho=2330 \mathrm{~kg} / \mathrm{m}^{3}, T_{0}=800 \mathrm{~K}, \\
& d_{n}=-9 \times 10^{-31} \mathrm{~m}^{3}, D_{E}=2.5 \times 10^{-3} \mathrm{~m}^{2} / \mathrm{s}, E_{g}=1.11 \mathrm{eV}, S_{0}=2 \mathrm{~m} / \mathrm{s}, \tau=5 \times 10^{-5} \mathrm{~s},
\end{aligned}
$$




$$
\begin{aligned}
& \alpha_{t_{1}}=0.04 \times 10^{-3} \mathrm{~K}^{-1}, \alpha_{t_{2}}=0.017 \times 10^{-3} \mathrm{~K}^{-1}, K=150 \mathrm{Wm}^{-1} \mathrm{~K}^{-1}, C_{e}=695 \mathrm{~J} /(\mathrm{kg} \mathrm{K}), \\
& j=0.2 \times 10^{-19} \mathrm{~m}^{2}, \gamma=0.779 \times 10^{-9} \mathrm{~N}, k=10^{10} \mathrm{Nm}^{-2}, t=0.001 \mathrm{~s}, \lambda_{0}=0.5 \times 10^{10} \mathrm{Nm}^{-2}, \\
& \lambda_{1}=0.5 \times 10^{10} \mathrm{Nm}^{-2}, \alpha_{0}=0.779 \times 10^{-9} \mathrm{~N}, \tau_{0}=0.00005 \mathrm{~s}, \quad v_{0}=0.0005 \mathrm{~s}, \quad n_{0}=10^{20} \mathrm{~m}^{-3} .
\end{aligned}
$$

The constants of Ge material is given in SI unit as [26, 27]:

$$
\begin{aligned}
& \lambda=0.48 \times 10^{11} \mathrm{~N} / \mathrm{m}^{2}, \mu=0.53 \times 10^{11} \mathrm{~N} / \mathrm{m}^{2}, \rho=5300 \mathrm{~kg} / \mathrm{m}^{3}, T_{0}=723 \mathrm{~K}, \tau=5 \times 10^{-5} \mathrm{~s}, \\
& d_{n}=-6 \times 10^{-31} \mathrm{~m}^{3}, D_{E}=10^{-2} \mathrm{~m}^{2} / \mathrm{s}, E_{g}=0.72 \mathrm{eV}, S_{0}=2 \mathrm{~m} / \mathrm{s}, n_{0}=10^{20} \mathrm{~m}^{-3}, \mathrm{k}=10^{10} \mathrm{Nm}^{-2} \\
& \alpha_{t_{1}}=6.1 \times 10^{-6} \mathrm{~K}^{-1}, \alpha_{t_{2}}=5.9 \times 10^{-6} \mathrm{~K}^{-1}, \mathrm{~K}=60 \mathrm{Wm}^{-1} \mathrm{~K}^{-1}, C_{e}=310 \mathrm{~J} /(\mathrm{kg} \mathrm{K}), j=0.2 \times 10^{-19} \mathrm{~m}^{2} \\
& , \gamma=0.779 \times 10^{-9} \mathrm{~N}, t=0.001 \mathrm{~s}, \lambda_{0}=0.5 \times 10^{10} \mathrm{Nm}^{-2}, \lambda_{1}=0.5 \times 10^{10} \mathrm{Nm}^{-2}, \alpha_{0}=0.779 \times 10^{-9} \mathrm{~N}, \\
& \tau_{0}=0.00005 \mathrm{~s}, \quad v_{0}=0.0005 \mathrm{~s} .
\end{aligned}
$$

The real part of the complex roots are used for carry out the numerical computational to obtain the wave distributions of the main quantities. In this section, the thermal, strain, mechanical and plasma waves can be obtained by temperature, displacement, stresses components and carrier density distributions. On the other hand, the microstretch distributions can be obtained by couple stress tensor component $m_{x y}$ and the first moment tensor (microstress) $\lambda_{z}$. All computational results are made against the horizontal distance $x$ inside the semiconductor medium at the plane $z=-2$. In this investigation the wave number and mechanical load are taken namely: $b=1$ and $\mathrm{P}^{*}=1$, in the general case of photothermo-microstretch elastic GPTMSE medium. For small value of $\zeta=0.05$ the complex constant $\omega$ can be taken in the form $\omega=\omega_{0}+i \zeta$, where $\omega_{0}=-2.5$ is real constants.

Figures 1 shows the distributions for silicon medium of the main physical quantities against the horizontal distance $x$ with the variation of three photothermoelasticity theories (CD, LS and GL models) according thermal memories (relaxation times). The wave distributions in figure 1 are obtained under the effect 
of rotation field when $\Omega=0.4$ in the context of GPTMSE. The wave distributions under the effect of different thermal relaxation times take the same behavior but they differ in the amplitude according to the value of relaxation times. All waves propagating through the medium satisfies the conditions that are applied to the surface. On the other hand, the wave propagation behavior for the CD, LS and GL models decrease and increase periodically in the same intervals until reaching equilibrium state when the distance tends to infinity.

Figures 2 studies the comparisons between the main non-dimensional physical fields $\left(T, u, \sigma_{z z}, N,, m_{x y}\right.$ and $\left.\lambda_{z}\right)$ against the horizontal distance under the impact of rotation $(\Omega=0.4)$ during the GPTMSE medium. The comparisons are carried out between the two samples of semiconductor, Si (solid lines) and $\mathrm{Ge}$ (dashed lines) materials at the same time under the same conditions when GL model is taken into accpunt. In this figure, the difference in the values of physical constants of $\mathrm{Si}$ and Ge materials tend to a greet significant influence on all the waves propagations.

Figures 3 displays the comparisons between the non-dimensional physical quantities $\left(T, u, \sigma_{z z}, N,, m_{x y}\right.$ and $\left.\lambda_{z}\right)$ against the horizontal distance under for silicon ( $\mathrm{Si}$ ) sample during the GPTMSE medium at the same time and the same conditions in GL model. The comparisons are made in two cases, the first case when the rotation parameter is neglected in the governing equations which it represented by solid lines with legend without rotation parameter. On the other hand, the second case when the medium is studied under the impact of rotation (dashed lines) which legend by with rotation parameter. In this figure, the impact of rotation on all physical distributions has a greet influence on the waves propagations. 
In the last figure, figure 4 shows the effect of microstretch parameters on all the physical distribution under investigation. The numerical calculations in this figure are made under the effect of rotation with GL model for Si material in two cases. The first case when the microstretch parameters are absent which represented by solid lines. However, the second case is obtained in the presence of microstretch parameters which represented by dashed lines. From this figure, the microstretch parameters have a great effect on all physical distribution.

\section{Conclusion}

The novel model of generalized thermal-microstretch during photothermal transport processes for an elastic semiconductor is investigated. The governing equations at a small time under the effect of rotation physical for $\mathrm{Si}$ and $\mathrm{Ge}$ media in vary photo-thermoelasticity theories according to thermal memories are obtained. From the above investigation, it is clear that:

- The thermal relaxation times in three models (CD, LS and GL) have a great impact on all wave propagations of the main physical fields under the influence of microstretch parameters and rotation.

- On the other hand, the different in the physical constants of two semiconductor materials (comparisons between $\mathrm{Si}$ and $\mathrm{Ge}$ ) have a great significant on all distributions.

- The impact of rotation plays an important rule in the generalized photothermo-microstretch theory theroticaly and graphically for Si material.

- Furthermore, the inner microstretch parameters of semiconductor elastic medium is more realistic than for that material without the microstretch parameters.

Many application can be used when the interaction between the thermal, plasma, microstretch and mechanical fields in elastic semicondactors are occurred. That 
applications can be obtained in modern aeronautics, astronautics, mechanical engineering and nuclear reactors.

\section{Appendix}

The basic coefficients of equation (38) are:

$$
\begin{gathered}
B_{1}=A_{2} A_{7}-a_{3} a_{7}-A_{1}-A_{3}-A_{4}-A_{6}-\alpha_{1}+\frac{a_{1} a_{11}-A_{5}}{a_{8}}, \\
B_{2}=\left(\frac{a_{1} a_{11}-A_{5}}{a_{8}}-A_{6}-A_{1}-b^{2}-\alpha_{1}-\frac{A_{5}}{a_{8}}\right) a_{3} a_{7}-\varepsilon_{4} A_{7} a_{4}+\left(\frac{\left(b^{2}+A_{3}+A_{4}+A_{6}+\alpha_{1}\right) a_{11}}{a_{8}}+\frac{c A_{7}}{a_{8}}\right) a_{1}-\frac{\omega a_{11} \varepsilon_{1} A_{2}}{a_{8}} \\
-a_{14} A_{9}+\left(A_{2} A_{4}+A_{2} A_{3}+\left(b^{2}+\alpha_{1}\right) A_{2}\right) A_{7}+\left(-A_{1}-A_{3}-A_{4}-\alpha_{1}\right) A_{6}+\left(-A_{3}-A_{1}-\alpha_{1}\right) A_{4} \\
\left(-A_{1}-\alpha_{1}\right) A_{3}-\alpha_{1} A_{3}+\varepsilon_{4} \varepsilon_{5}+\frac{A_{2} A_{5} A_{7}-A_{5} A_{6}+\left(-A_{1}-A_{3}-A_{4}-\alpha_{1}\right) A_{5}-c \omega \varepsilon_{1}}{a_{8}}
\end{gathered}
$$

$$
\begin{aligned}
& \left.B_{3}=-\varepsilon_{4} A_{7} a_{3} a_{4}+\left(-\left(2 b^{2}-\alpha_{1}\right) A_{2} A_{7}+\left(-b^{2}-A_{1}-\alpha_{1}\right) A_{6}-\left(b^{2}+\alpha_{1}\right) A_{1}-b^{2} \alpha_{1}+\varepsilon_{4} \varepsilon_{5}\right) a_{3}\right) a_{7} \\
& +\left(-b^{2} \varepsilon_{4}-A_{3} \varepsilon_{4}-A_{4} \varepsilon_{4}\right) A_{7} a_{4}+\left(-A_{6}-A_{4}-\alpha_{1}\right) A_{9} a_{14}+ \\
& \left(\left(A_{2} A_{3}-\left(-b^{2}-\alpha_{1}\right) A_{2} A_{3}+b^{2} \alpha_{1} A_{2}\right) A_{7}+\left(\left(-A_{3}-A_{1}-\alpha_{1}\right) A_{4}\right.\right. \\
& \left.+\left(-A_{1}-\alpha_{1}\right) A_{3}-\alpha_{1} A_{1}\right) A_{6}+\left(\left(-A_{1}-\alpha_{1}\right) A_{3}-\alpha_{1} A_{1}+\varepsilon_{4} \varepsilon_{5}\right) A_{4}+\left(-\alpha_{1} A_{1}+\varepsilon_{4} \varepsilon_{5}\right) A_{3}+\varepsilon_{4} \varepsilon_{5} A_{1} \\
& +\frac{1}{a_{8}}\left(\begin{array}{l}
\left(\left(\left(2 b^{2}+A_{6}+\alpha_{1}\right) a_{11}+c A_{7}\right) a_{1}-\omega \varepsilon_{1} A_{2} a_{11}+A_{2} A_{5} A_{7}-A_{5} A_{6}+\left(-b^{2}-A_{1}-\alpha_{1}\right) A_{5}-\omega c \varepsilon_{1}\right)+\left(\omega a_{11} \varepsilon_{1} \varepsilon_{4}-A_{5} A_{7} \varepsilon_{4}\right) a_{4} \\
+\left(\left(\left(b^{2}+A_{3}+A_{4}+\alpha_{1}\right) A_{6}+\left(b^{2}+A_{3}+\alpha_{1}\right) A_{4}-\left(-b^{2}-\alpha_{1}\right) A_{3}+b^{2} \alpha_{1}-\varepsilon_{4} \varepsilon_{5}\right) a_{11}+\left(b^{2} c+c A_{3}+c A_{4}+c \alpha_{1}\right) A_{7}\right) a_{1}
\end{array}\right), \\
& -A_{5} A_{9} a_{14}+\left(-\omega \varepsilon_{1} A_{2} A_{4}-\omega \varepsilon_{1} A_{2} A_{3}-\left(b^{2} \omega \varepsilon_{1}+\omega \alpha_{1} \varepsilon_{1}\right) A_{2}\right) a_{11}+\left(A_{2} A_{4}+A_{2} A_{3}-\left(-b^{2}-\alpha_{1}\right) A_{2}\right) A_{5} A_{7}+\left(-A_{4}-A_{3}-A_{1}-\alpha_{1}\right) \\
& A_{5} A_{6}+\left(\left(-A_{3}-A_{1}-\alpha_{1}\right) A_{4}+\left(-A_{1}-\alpha_{1}\right) A_{3}-\alpha_{1} A_{1}+\varepsilon_{4} \varepsilon_{5}\right) A_{5}-c \varepsilon_{1} A_{4} \\
& \left.-c \varepsilon_{1} \omega A_{3}-c \varepsilon_{1} \omega A_{1}-c \omega \varepsilon_{1} \alpha_{1}\right)
\end{aligned}
$$




$$
\begin{aligned}
& B_{4}=\left(\left(\left(b^{4}+2 b^{2} \alpha_{1}\right) A_{2}-2 b^{2} a_{4} \varepsilon_{4}\right) A_{7}+\left(\left(-b^{2}-\alpha_{1}\right) A_{1}-b^{2} \alpha_{1}\right) A_{6}\right. \\
& \left.+\left(-b^{2} \alpha_{1}+\varepsilon_{4} \varepsilon_{5}\right) A_{1}+b^{2} \varepsilon_{4} \varepsilon_{5}\right) a_{3} a_{7}+\left(\left(-A_{4}-\alpha_{1}\right) A_{6}-A_{4} \alpha_{1}+\varepsilon_{4} \varepsilon_{5}\right) A_{9} a_{14}+ \\
& \left(\left(\left(\left(b^{2}+\alpha_{1}\right) A_{2}-a_{4} \varepsilon_{2}\right) A_{3}+b^{2} \alpha_{1} A_{2}-b^{2} a_{4} \varepsilon_{4}\right) A_{4}+\left(\alpha_{1} b^{2} A_{2}-b^{2} a_{4} \varepsilon_{4}\right) A_{3}\right) A_{7} \\
& +\left(\left(\left(-A_{1}-\alpha_{1}\right) A_{3}-A_{1} \alpha_{1}\right) A_{4}-A_{1} \alpha_{1} A_{3}\right) A_{6}+\left(\left(-A_{1} \alpha_{1}+\varepsilon_{4} \varepsilon_{5}\right) A_{3}+\varepsilon_{4} \varepsilon_{5} A_{1}\right) A_{4}+\varepsilon_{4} \varepsilon_{5} A_{1} A_{3}+ \\
& \frac{1}{a_{8}}\left(\left(\left(\left(2 b^{2}+\alpha_{1}\right) A_{6}+b^{4}+2 b^{2} \alpha_{1}-\varepsilon_{4} \varepsilon_{5}\right) a_{11}+\left(2 b^{2} c+c \alpha_{1}\right) A_{7}\right) a_{1}+\left(\left(-2 b^{2} \omega \varepsilon_{1}-\omega \alpha_{1} \varepsilon_{1}\right) A_{2}+\omega a_{4} \varepsilon_{1} \varepsilon_{4}\right)\right. \\
& +\left(\left(2 b^{2}+\alpha_{1}\right) A_{2}-a_{4} \varepsilon_{4}\right) A_{5} A_{7}+\left(-b^{2}-A_{1}-\alpha_{1}\right) A_{5} A_{6}+\left(\left(-b^{2}-\alpha_{1}\right) A_{1}-b^{2} \alpha_{1}+\varepsilon_{4} \varepsilon_{5}\right) A_{5} \\
& \left.-c \omega \varepsilon_{1} A_{1}-c b^{2} \omega \varepsilon_{1}-c \omega \alpha_{1} \varepsilon_{1}\right) a_{3} a_{7}+\left(\left(\left(\left(b^{2}+A_{3}+\alpha_{1}\right) A_{4}+\left(b^{2}+\alpha_{1}\right) A_{3}+b^{2} \alpha_{1}\right) A_{6}\right.\right. \\
& \left.\left.+\left(\left(b^{2}+\alpha_{1}\right) A_{3}+b^{2} \alpha_{1}-\varepsilon_{4} \varepsilon_{5}\right) A_{4}+\left(b^{2} \alpha_{1}-\varepsilon_{4} \varepsilon_{5}\right) A_{3}\right)-b^{2} \varepsilon_{4} \varepsilon_{5}\right) a_{11}+\left(\left(b^{2} c+c A_{3}+\alpha_{1} c\right) A_{4}\right. \\
& \left.\left.+\left(b^{2} c+c \alpha_{1}\right) A_{3}+b^{2} c \alpha_{1}\right) A_{7}\right) a_{1}+\left(-A_{5} A_{6}+\left(-A_{4}-\alpha_{1}\right) A_{5}-c \omega \varepsilon_{1}\right) A_{9} a_{14} \\
& +\left(\left(-\omega \varepsilon_{1} A_{2} A_{3}+\left(-b^{2} \omega \varepsilon_{1}-\alpha_{1} \omega \varepsilon_{1}\right) A_{2}+\omega \varepsilon_{1} \varepsilon_{4}\right) A_{4}+\left(\left(-\omega b^{2} \varepsilon_{1}-\omega \alpha_{1} \varepsilon_{1}\right) A_{2}+\omega a_{4} \varepsilon_{1} \varepsilon_{4}\right) A_{3}\right. \\
& \left.-b^{2} \alpha_{1} \omega \varepsilon_{1} A_{2}+b^{2} a_{4} \omega \varepsilon_{1} \varepsilon_{4}\right) a_{11}+\left(\left(A_{2} A_{3}+\left(b+\alpha_{1}\right) A_{2}-a_{4} \varepsilon_{1}\right) A_{4}+\varepsilon_{1} \varepsilon_{4}+\left(\left(b^{2}+\alpha_{1}\right) A_{2}-a_{4} \varepsilon_{4}\right) A_{3}\right. \\
& \left.+b^{2} \alpha_{1} A_{2}-b^{2} a_{4} \varepsilon_{4}\right) A_{5} A_{7}+\left(\left(-A_{3}-A_{1}-\alpha_{1}\right) A_{4}+\left(-A_{1}-\alpha_{1}\right) A_{3}-A_{1} \alpha_{1}\right) A_{5} A_{6}+ \\
& \left(\left(\left(-A_{1}-\alpha_{1}\right) A_{3}-A_{1} \alpha_{1}+\varepsilon_{4} \varepsilon_{5}\right) A_{4}+\left(-A_{1} \alpha_{1}+\varepsilon_{4} \varepsilon_{5}\right) A_{3}+\varepsilon_{4} \varepsilon_{5} A_{1}\right. \\
& ) A_{5}+\left(-c \omega A_{1} \varepsilon_{1}-c \omega A_{3} \varepsilon_{1}-c \omega \alpha_{1} \varepsilon_{1}\right) A_{4}+\left(-c \omega A_{1} \varepsilon_{1}-c \omega \alpha_{1} \varepsilon_{1}\right) A_{3}-c \omega \alpha_{1} \varepsilon_{1} A_{1}\right) \\
& B_{5}=\left(-b^{4} \varepsilon_{4} A_{7} a_{3} a_{4}+\left(b^{4} A_{2} A_{7} \alpha_{1}-b^{2} A_{1} A_{6}+b^{2} A_{1} \varepsilon_{4} \varepsilon_{5}\right) a_{3}\right) a_{7}-b^{2} A_{3} A_{4} A_{7} \varepsilon_{4} a_{4} \\
& +\left(-A_{4} A_{6} \alpha_{1}+A_{4} \varepsilon_{4} \varepsilon_{5}\right) A_{9} a_{14}+b^{2} \alpha_{1} A_{2} A_{3} A_{4} A_{7}-\alpha_{1} A_{1} A_{3} A_{4} A_{6}+\varepsilon_{4} \varepsilon_{5} A_{1} A_{3} A_{4} \\
& +\frac{1}{a_{8}}\left(\left(\left(2 b^{2} \omega a_{11} \varepsilon_{1} \varepsilon_{4}-2 b^{2} A_{5} A_{7} \varepsilon_{4}\right) a_{3} a_{4}+\left(\left(\left(-\left(-b^{4}-2 b^{2} \alpha_{1}\right) A_{6}+b^{4} \alpha_{1}-2 b^{2} \varepsilon_{4} \varepsilon_{5}\right) a_{11}\right.\right.\right.\right. \\
& \left.-\left(-b^{4} c-2 b^{2} c \alpha_{1}\right) A_{7}\right) a_{1}-\left(b^{4} \omega \varepsilon_{1}+2 b^{2} \alpha_{1} \omega \varepsilon_{1}\right) A_{2} a_{11}-\left(-b^{4}-2 b^{2} \alpha_{1}\right) A_{2} A_{5} A_{7} \\
& +\left(-\left(b^{2}+\alpha_{1}\right) A_{1}-b^{2}\right) A_{5} A_{6}+\left(-\left(b^{2}-\varepsilon_{4} \varepsilon_{5}\right) A_{1}+b^{2} \varepsilon_{4} \varepsilon_{5}\right) A_{5}-\left(b^{2} c \omega \varepsilon_{1}+c \alpha_{1} \omega \varepsilon_{1}\right) A_{1} \\
& \left.\left.-b^{2} c \alpha_{1} \omega \varepsilon_{1}\right) a_{3}\right) a_{7}+\left(\left(\left(b^{2} \omega \varepsilon_{1} \varepsilon_{4}+A_{3} \omega \varepsilon_{1} \varepsilon_{4}\right) A_{4}+b^{2} \varepsilon_{1} \varepsilon_{4} A_{3}\right) a_{11}+\left(\left(-b^{2} \varepsilon_{4}-A_{3} \varepsilon_{4}\right) A_{4}\right.\right. \\
& \left.\left.-b^{2} \varepsilon_{4} A_{3}\right) A_{5} A_{7}\right) a_{4}+\left(\left(\left(\left(-\left(-b^{2}-\alpha_{1}\right) A_{3}+b^{2} \alpha_{1}\right) A_{4}+b^{2} \alpha_{1} A_{3}\right) A_{6}+\left(-\left(b^{2} \alpha_{1}+\varepsilon_{4} \varepsilon_{5}\right) A_{3}\right.\right.\right. \\
& \left.\left.\left.-b^{2} \varepsilon_{4} \varepsilon_{5}\right) A_{4}-b^{2} \varepsilon_{4} \varepsilon_{5} A_{3}\right) a_{11}+\left(\left(-\left(b^{2} c-c \alpha_{1}\right) A_{3}+b^{2} \alpha_{1} c\right) A_{4}+b^{2} c \alpha_{1} A_{3}\right) A_{7}\right) a_{1}+\left(\left(-A_{4}-\alpha_{1}\right) A_{5} A_{6}\right. \\
& \left.+\left(-A_{4} \alpha_{1}+\varepsilon_{4} \varepsilon_{5}\right) A_{5}-c \omega \varepsilon_{1} A_{4}-c \alpha_{1} \omega \varepsilon_{1}\right) A_{9} a_{14}+\left(\left(-\left(b^{2} \omega \varepsilon_{1}+\alpha_{1} \omega \varepsilon_{1}\right) A_{2} A_{3}-b^{2} \alpha_{1} \omega \varepsilon_{1} A_{2}\right) A_{4}\right. \\
& \left.\left.-b^{2} \varepsilon_{1} \alpha_{1} \omega A_{2} A_{3}\right) a_{11}+\left(-\left(-b^{2}-\alpha_{1}\right) A_{2} A_{3}+b^{2} \alpha_{1} A_{2}\right) A_{4}+b^{2} \alpha_{1} A_{2} A_{3}\right) A_{5} A_{7}+\left(\left(\left(-A_{1}-\alpha_{1}\right) A_{3}\right.\right. \\
& \left.\left.-\alpha_{1} A_{1}\right) A_{4}-\alpha_{1} A_{1} A_{3}\right) A_{5} A_{6}+\left(\left(\left(-A_{1} \alpha_{1}+\varepsilon_{4} \varepsilon_{5}\right) A_{3}+\varepsilon_{4} \varepsilon_{5} A_{1}\right) A_{4}+\varepsilon_{4} \varepsilon_{5} A_{1} A_{3}\right) A_{5}+\left(\left(-c \omega \varepsilon_{1} A_{1}-c \omega \alpha_{1} \varepsilon_{1}\right) A_{3}\right. \\
& \left.\left.-c \omega \alpha_{1} \varepsilon_{1} A_{1}\right) A_{4}-c \omega \alpha_{1} \varepsilon_{1} A_{1} A_{3}\right)
\end{aligned}
$$




$$
\begin{aligned}
& B_{6}=\frac{1}{a_{8}}\left(\left(\left(b^{4} \varepsilon_{4}\left(\omega a_{11} \varepsilon_{1}-A_{5} A_{7} \varepsilon_{4}\right)+\left(\left(\left(b^{4} a_{11}\left(A_{6} \alpha_{1}-\varepsilon_{4} \varepsilon_{5}\right)+b^{2} c \alpha_{1} A_{7}\right) a_{1}\right.\right.\right.\right.\right. \\
& \left.\left.-b^{4} \omega \alpha_{1} \varepsilon_{1} A_{2} a_{11}+b^{4} \alpha_{1} A_{2} A_{5} A_{7}-b^{2} \alpha_{1} A_{1} A_{5} A_{6}+b^{2} \varepsilon_{4} \varepsilon_{5} A_{1} A_{5}-b^{2} c \omega \alpha_{1} \varepsilon_{1} A_{1}\right) a_{3}\right) a_{7} \\
& +\left(b^{2} \omega A_{3} A_{4} \varepsilon_{1} \varepsilon_{4}-b^{2} A_{3} A_{4} A_{5} A_{7} \varepsilon_{4}\right)+\left(\left(b^{2} A_{3} A_{4} A_{6} \alpha_{1}-b^{2} A_{3} A_{4} \varepsilon_{4} \varepsilon_{5}\right) a_{11}+b^{2} \alpha_{1} c A_{3} A_{4} A_{7}\right) a_{1} . \\
& +\left(-c \omega A_{4} \alpha_{1} \varepsilon_{1}-A_{4} A_{5} A_{6} \alpha_{1}+A_{4} A_{5} \varepsilon_{4} \varepsilon_{5}\right) A_{9} a_{14}-b^{2} \alpha_{1} A_{2} \varepsilon_{1} A_{3} A_{4} a_{11}+b^{2} \alpha_{1} A_{2} A_{3} A_{4} A_{5} A_{7} \\
& \left.-\alpha_{1} A_{1} A_{3} A_{4} A_{5} A_{6}+\varepsilon_{4} \varepsilon_{5} A_{1} A_{3} A_{4} A_{5}-c \omega \varepsilon_{1} \alpha_{1} A_{1} A_{3} A_{4}\right)
\end{aligned}
$$

On the other hand, the basic coefficients of equation (47) are:

$$
\begin{gathered}
E_{1}=\frac{\left(\begin{array}{c}
\left(\varepsilon_{1} \omega+A_{7}\left(a_{8} k_{n}^{2}-A_{5}\right)\right)\left(k_{n}^{2}-b^{2}\right)\left(\left(k_{n}^{2}-A_{6}\right)\left(k_{n}^{2}-\alpha_{1}\right)-\varepsilon_{4} \varepsilon_{5}\right)- \\
A_{7}\left(k_{n}^{2}-b^{2}\right)\left(k_{n}^{2}-\alpha_{1}\right)\left[\varepsilon_{1} \omega A_{8}-\varepsilon_{4} \varepsilon_{5}+\left(k_{n}^{2}-A_{6}\right)\left(a_{8} k_{n}^{2}-A_{5}\right)\right]
\end{array}\right)}{\left(k_{n}^{2}-\alpha_{1}\right)\left(\varepsilon_{1} \omega A_{9}+\left(k_{n}^{2}-A_{6}\right)\left(a_{8} k_{n}^{2}-A_{5}\right)\right)-\varepsilon_{4} \varepsilon_{5}}, \\
E_{2}=\frac{\left(\left(\varepsilon_{1} \omega+A_{7}\left(a_{8} k_{n}^{2}-A_{5}\right)\right)\left(k_{n}^{2}-b^{2}\right)\left(k_{n}^{2}-\alpha_{1}\right)\right)}{\left(k_{n}^{2}-\alpha_{1}\right)\left(\varepsilon_{1} \omega A_{9}+\left(k_{n}^{2}-A_{6}\right)\left(a_{8} k_{n}^{2}-A_{5}\right)\right)-\varepsilon_{4} \varepsilon_{5}}, \\
E_{3}=\frac{\varepsilon_{4}\left(\left(\varepsilon_{1} \omega+A_{7}\left(a_{8} k_{n}^{2}-A_{5}\right)\right)\left(k_{n}^{2}-b^{2}\right)\right)}{\varepsilon_{4} \varepsilon_{5}-\left(k_{n}^{2}-\alpha_{1}\right)\left(\varepsilon_{1} \omega A_{9}+\left(k_{n}^{2}-A_{6}\right)\left(a_{8} k_{n}^{2}-A_{5}\right)\right)}, \\
E_{4}=\frac{\left.a_{14}\left(k_{n}^{2}-A_{4}\right)\right)}{k_{n}^{4}-\left(a_{3} a_{7}+A_{3}+A_{7}\right) k_{n}^{2}+\left(A_{3} A_{4}+b^{2} a_{3} a_{7}\right)}, \\
E_{5}=\frac{\left.a_{7} a_{14}\left(k_{n}^{2}-b^{2}\right)\right)}{k_{n}^{4}-\left(a_{3} a_{7}+A_{3}+A_{7}\right) k_{n}^{2}+\left(A_{3} A_{4}+b^{2} a_{3} a_{7}\right)} .
\end{gathered}
$$

The main parameters of equations (52) can be obtained as:

$$
\left.\begin{array}{cc}
s_{1}=f_{1} k_{1}, \quad s_{2}=f_{1} k_{2}, & s_{3}=f_{2}+\lambda k_{3}^{2}-f_{3}, \quad s_{4}=f_{2}+\lambda k_{4}^{2}-f_{3}, \\
s_{5}=f_{2}+\lambda k_{5}^{2}-f_{3}, & s_{6}=f_{2}+\lambda k_{6}^{2}-f_{3}, \quad f_{5}=b^{2} \mu . \\
r_{1}=f_{5}+f_{4} k_{1}^{2}+k, & r_{2}=f_{5}+f_{4} k_{2}^{2}+k, \quad r_{3}=f_{1} k_{3}, \\
r_{4}=f_{1} k_{4}, \quad r_{5}=f_{1} k_{5}, & r_{6}=f_{1} k_{6}, f_{1}=-i b(2 \mu+k), \\
\wedge & \wedge \\
f_{2}=\lambda_{0}-b^{2}(\lambda+2 \mu+k), f_{3}=\gamma\left(1+d_{n}\right), f_{4}=\mu+k .
\end{array}\right\}
$$

\section{References}

[1] J. C. Misra, N. C. Chattopadhyay, and S. C. Samanta, Study of the thermoelastic interactions in an elastic half space subjected to a ramp-type heating - A state-space approach, Int. J. Eng. Sci., 34(5), 579-596, (1996). 
[2] Eringen, A. Cemal, Linear theory of micropolar elasticity, J. Math. Mech., 909923, (1966).

[3] Eringen, A. Cemal, Theory of thermo-microstretch elastic solids, Int. J. Eng. Sci., 28(12), 1291-1301, (1990).

[4]Singh, Baljeet, Reflection and refraction of plane waves at a liquid/thermomicrostretch elastic solid interface, Int. J. Eng. Sci., 39(5), 583-598, (2001).

[5] M. Othman and Kh. Lotfy, The influence of gravity on 2-D problem of two temperature generalized thermoelastic medium with thermal relaxation. Journal of Computational and Theoretical Nanoscience, 12, 2587-2600, (2015).

[6] De Cicco and L. Nappa, On the theory of thermomicrostretch elastic solids, J. Therm. Stress., 22(6), 565-580, (1999).

[7] M. Othman and Kh Lotfy, On the plane waves of generalized thermomicrostretch elastic half-space under three theories, Int. Comm. Heat and Mass Trans., 37(2), 192-200, (2010).

[8] Kh Lotfy and S. M. Abo-Dahab, Two-dimensional problem of two temperature generalized thermoelasticity with normal mode analysis under thermal shock problem, Journal of Computational and Theoretical Nanoscience, 12(8), 1709-1719, (2015).

[9] M. Othman and Kh. Lotfy, Effect of rotating on plane waves in generalized thermo-microstretch elastic solid with one relaxation time, Multidiscipline Modeling in Mat. and Str., 7 (1), 43-62, (2011).

[10] G. Ramesh, B. Prasannakumara, B. Gireesha and M. Rashidi, Casson fluid flow near the stagnation point over a stretching sheet with variable thickness and radiation, Journal of Applied Fluid Mechanics, 9(3), 1115-1122, (2016). 
[11] M. Ezzat and M. Abd-Elaal, Free convection effects on a viscoelastic boundary layer flow with one relaxation time through a porous medium, Journal of the Franklin Institute, 334, (4), 685-706, (1997).

[12] M. Ezzat and M. Abd-Elaal, State Space Approach to Viscoelastic Fluid Flow of Hydromagnetic Fluctuating Boundary-Layer through a Porous Medium, Z. angew. Math. Mech., 77, 197-207, (1997).

[13] M. Ezzat, Free convection effects on perfectly conducting fluid, International Journal of Engineering Science, 39(7), 799-819, (2001).

[14] E. Carrera and S. Valvano, A variable ESL/LW kinematic plate formulation for free-vibration thermoelastic analysis of laminated structures, Journal of Thermal Stresses, 42(4), 452-474, (2019).

[15] E. Carrera and S. Valvano, A variable kinematic shell formulation applied to thermal stress of laminated structures, Journal of Thermal Stresses ,40(7), 803-827, (2017).

[16] M. Cinefra, S. Valvano and E. Carrera, Thermal stress analysis of laminated structures by a variable kinematic MITC9 shell element, Journal of Thermal Stresses, 39(2), 121-141, (2016).

[17] M. Cinefra, S. Valvano and E. Carrera, Heat conduction and Thermal Stress Analysis of laminated composites by a variable kinematic MITC9 shell element, Curved and Layered Structures 2: 301-320, (2015).

[18] J. P. Gordon, R.C.C. Leite, R.S. Moore, S.P.S.Porto, J.R.Whinnery, Longtransient effects in lasers with inserted liquid samples. Bull. Am. Phys. Soc. 119, 501-510, (1964).

[19] L.B. Kreuzer, Ultralow gas concentration infrared absorption spectroscopy. J. Appl. Phys. 42, 2934 (1971).

[20] A.C. Tam, Ultrasensitive Laser Spectroscopy, pp. 1-108. Academic Press, New York (1983). 
[21] A.C. Tam, Applications of photoacoustic sensing techniques. Rev. Mod. Phys. 58, 381 (1986).

[22] A.C. Tam, Photothermal Investigations in Solids and Fluids, pp. 1-33. Academic Press, Boston (1989)

[23]]A. Hobinya, I. Abbas, A GN model on photothermal interactions in a twodimensions semiconductor half space, Results in Physics, 15, 102588, (2019).

[24] D.M. Todorovic, P.M. Nikolic, A.I. Bojicic, Photoacoustic frequency transmission technique: electronic deformation mechanism in semiconductors. J. Appl. Phys. 85, 7716 (1999).

[25] Y.Q. Song, D.M. Todorovic, B. Cretin, P. Vairac, Study on the generalized thermoelastic vibration of the optically excited semiconducting microcantilevers. Int. J. Solids Struct. 47, 1871 (2010).

[26] Kh. Lotfy, The elastic wave motions for a photothermal medium of a dualphase-lag model with an internal heat source and gravitational field. Can J. Phys., 94,400-409 (2016).

[27] Kh. Lotfy, A Novel Model of Photothermal Diffusion (PTD) fo Polymer Nano- composite Semiconducting of Thin Circular Plate . Physica B- Condenced Matter, 537, 320-328 (2018).

[28] Kh. Lotfy, R. Kumar, W. Hassan and M. Gabr. Thermomagnetic effect with microtemperature in a semiconducting Photothermal excitation medium Appl. Math. Mech. Engl. Ed., 39(6), 783-796(2018).

[29] Kh. Lotfy and M. Gabr, Response of a semiconducting infinite medium under two temperature theory with photothermal excitation due to laser pulses, Optics and Laser Technology, 97, 198-208 (2017).

[30] Kh. Lotfy, Photothermal waves for two temperature with a semiconducting medium under using a dual-phase-lag model and hydrostatic initial stress, Waves in Random and Complex Media, 27(3), 482-501(2017).

[31] Kh. Lotfy, A novel model for Photothermal excitation of variable thermal conductivity semiconductor elastic medium subjected to mechanical ramp type with two-temperature theory and magnetic field. Sci. Rep., 9, ID 3319 (2019). 
[32] Kh. Lotfy, Effect of Variable Thermal Conductivity during the Photothermal Diffusion Process of Semiconductor Medium, Silicon, 11, 1863-1873 (2019).

[33] I. Abbas, F. Alzahranib and A. Elaiwb, A DPL model of photothermal interaction in a semiconductor material, Waves in Random and Complex media, 29 , 328-343, (2019).

[34] A. Khamis, A. El-Bary, Kh. Lotfy and A. Bakali, Photothermal excitation processes with refined multi dual phase-lags theory for semiconductor elastic medium, Alex. Eng. J. 59 (1), 1-9, ( 2020).

[35] Kh. Lotfy, A. El-Bary and A. El-Sharif, Ramp-type heating micro-temperature for a rotator semiconducting material during photo-excited processes with magnetic field, Results in Phys. 19, 103338, (2020).

[36] Kh. Lotfy, Effect of variable thermal conductivity and rotation of semiconductor elastic medium through two-temperature photothermal excitation, Waves in Random and Complex Media, doi: 10.1080/17455030.2019.1588483, (2019).

[37] Kh. Lotfy, R. Tantawi and N. Anwer, Response of Semiconductor Medium of Variable Thermal Conductivity Due to Laser Pulses with Two-Temperature through Photothermal Process, Silicon, 11, 2719-2730, (2019).

[38] M. Yasein, N. Mabrouk, Kh. Lotfy and A. EL-Bary, The influence of variable thermal conductivity of semiconductor elastic medium during photothermal excitation subjected to thermal ramp type, Results in Phys., 15, 102766, (2019).

[39] S. Mondal and A. Sur, Photo-thermo-elastic wave propagation in an orthotropic semiconductor with a spherical cavity and memory responses, Waves in Random and Complex Media, doi: 10.1080/17455030.2019.1705426, (2020).

[40] M. Ezzat, Hyperbolic thermal-plasma wave propagation in semiconductor of organic material, Waves in Random and complex Media, doi: 10.1080/17455030. 2020.1772524, (2020).

[41] M. Ezzat, A novel model of fractional thermal and plasma transfer within a non-metallic plate, Smart Structures and Systems, 27(1), 73-87, (2021). 
[42] H. Lord and Y. Shulman, A generalized dynamical theory of thermoelasticity. J Mech Phys Solid., 15, 299-309, (1967).

[43] A. Green and K. Lindsay, Thermoelasticity. J Elast., 2, 1-7, (1972).

[44] M. Biot, Thermoelasticity and irreversible thermodynamics. J Appl Phys., 27, 240-253, (1956).

[45] H. Deresiewicz, Plane waves in a thermoelastic solid, J. Acoust. Soc. Am., 29 204-209, (1957).

[46] P. Chadwick, I.N. Sneddon, Plane waves in an elastic solid conducting heat, J. Mech. Phys. Solids, 6, 223-230, (1958).

[47] P. Chadwick, Thermoelasticity: the dynamic theory, in: R. Hill, I.N. Sneddon (Eds.), Progress in Solid Mechanics, vol. I, North-Holland, Amsterdam, 263-328, (1960).

[48]Todorović DM, Nikolić PM, Bojičić AI. Photoacoustic frequency transmission technique: electronic deformation mechanism in semiconductors. J. Appl. Phys., 85, 7716-7726, (1999).

[49] Mandelis, A., Nestoros, M., Christofides, C.: Thermoelectronic-wave coupling in laser photothermal theory of semiconductors at elevated temperatures. Opt. Eng., 36 (2), 459-468, (1997).

[50] Kh. Lotfy, S. M. Abo-Dahab, R. Tantawi and N. Anwer, Thermomechanical Response Model of a Reflection Photo thermal Diffusion Waves (RPTD) for Semiconductor Medium, Silicon, 12(1), 199-209, (2020).

[51] Kh. Lotfy , W. Hassan, A.A. El-Bary and Mona A. Kadry, Response of electromagnetic and Thomson effect of semiconductor mediu due to laser pulses and thermal memories during photothermal excitation, Results in Physics, 16, 102877, (2020). 

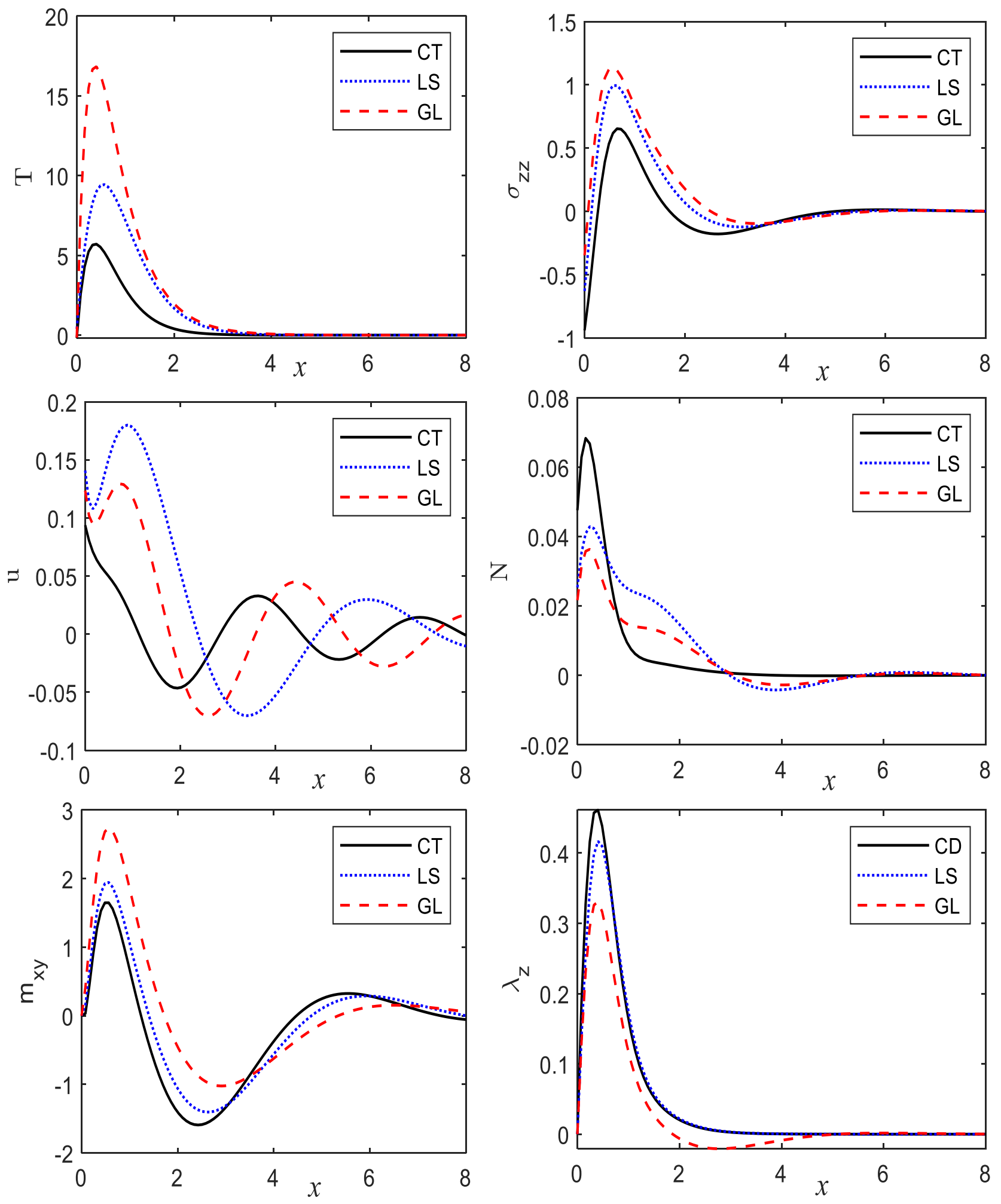

Figure 1 The variation of the main fields against the horizontal distance of $\mathrm{Si}$ medium under the influence of three different theories according to the thermal relaxation times. 

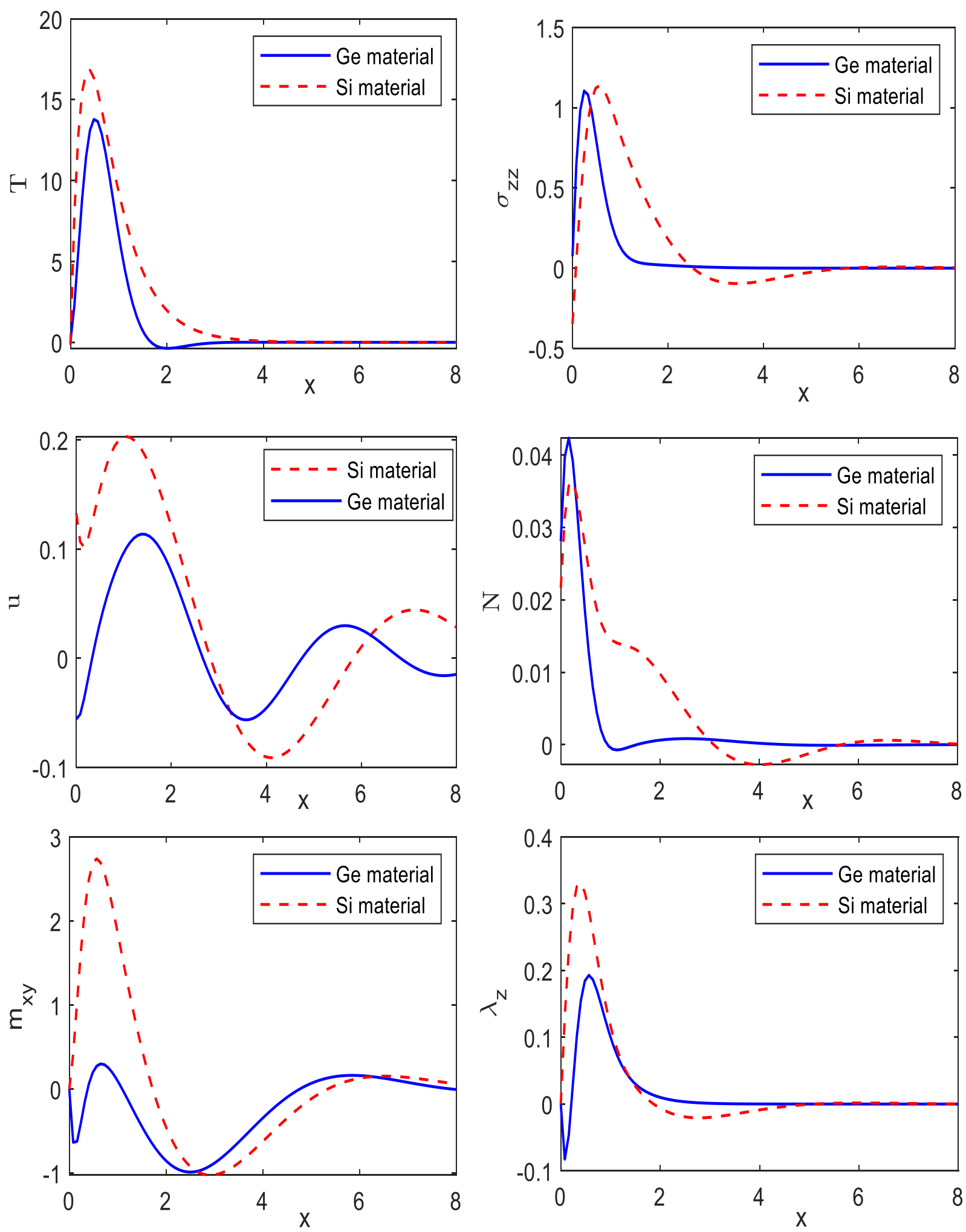

Figure 2 The comparison between $\mathrm{Si}$ and Ge materials of the main physical fields against distance in the generalized GL theory. 

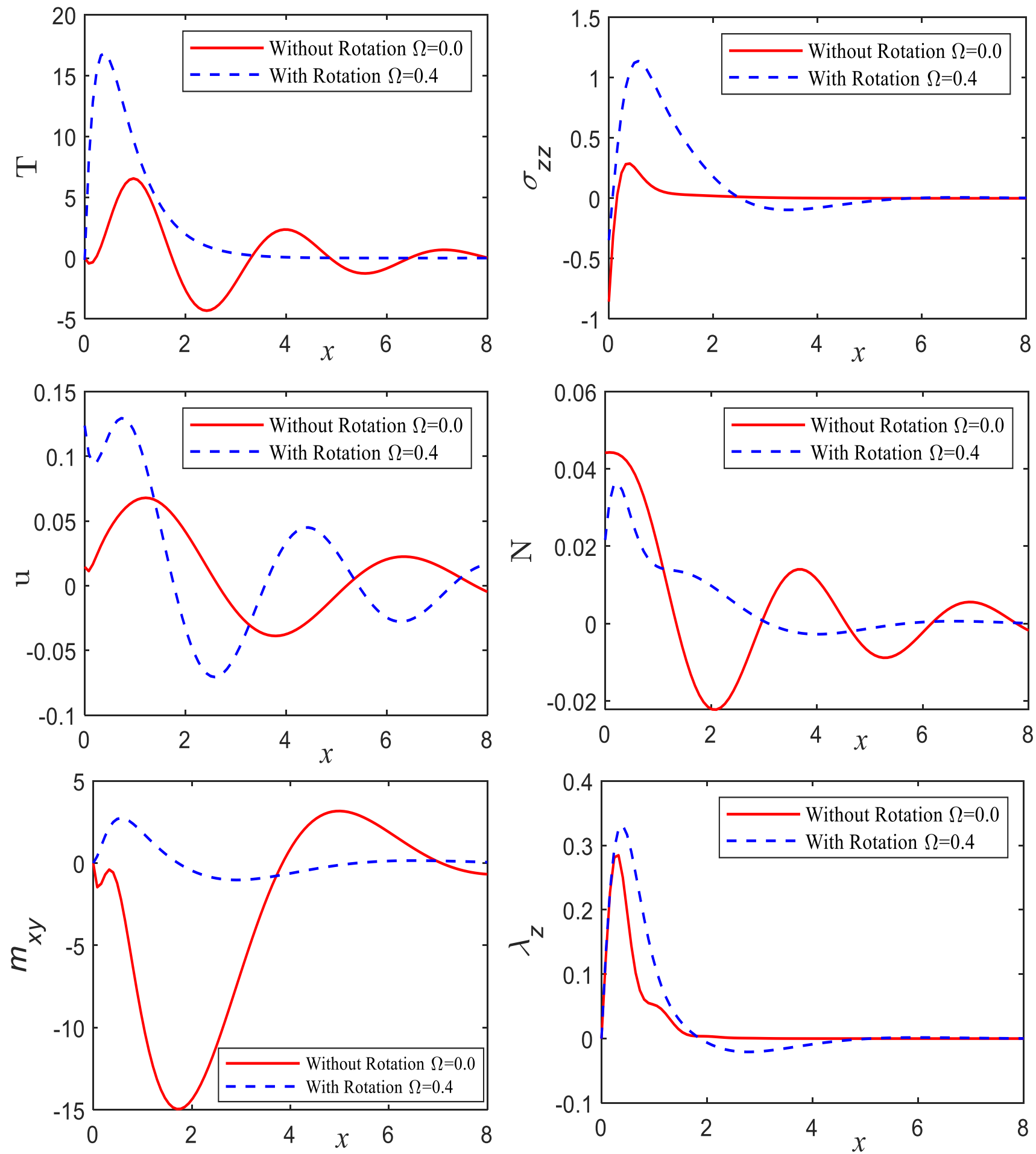

Figure 3 The comparison between the main physical fields against the horizontal distance under the effect of rotation parameters in the generalized GL theory of Si medium. 

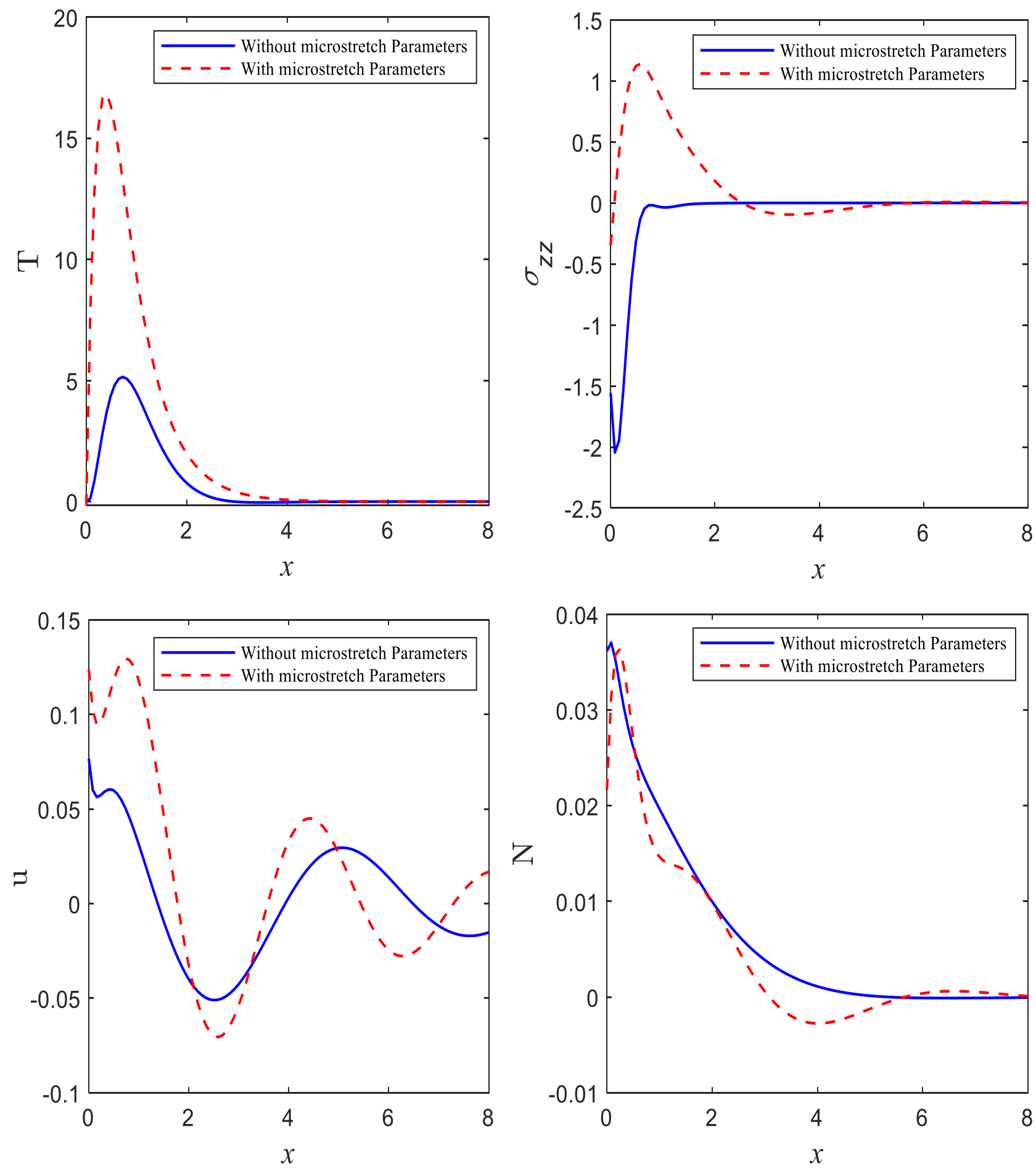

Figure 4 The comparison between the main physical fields against the horizontal distance under the effect of microstretch parameters in the generalized GL theory of Si medium. 

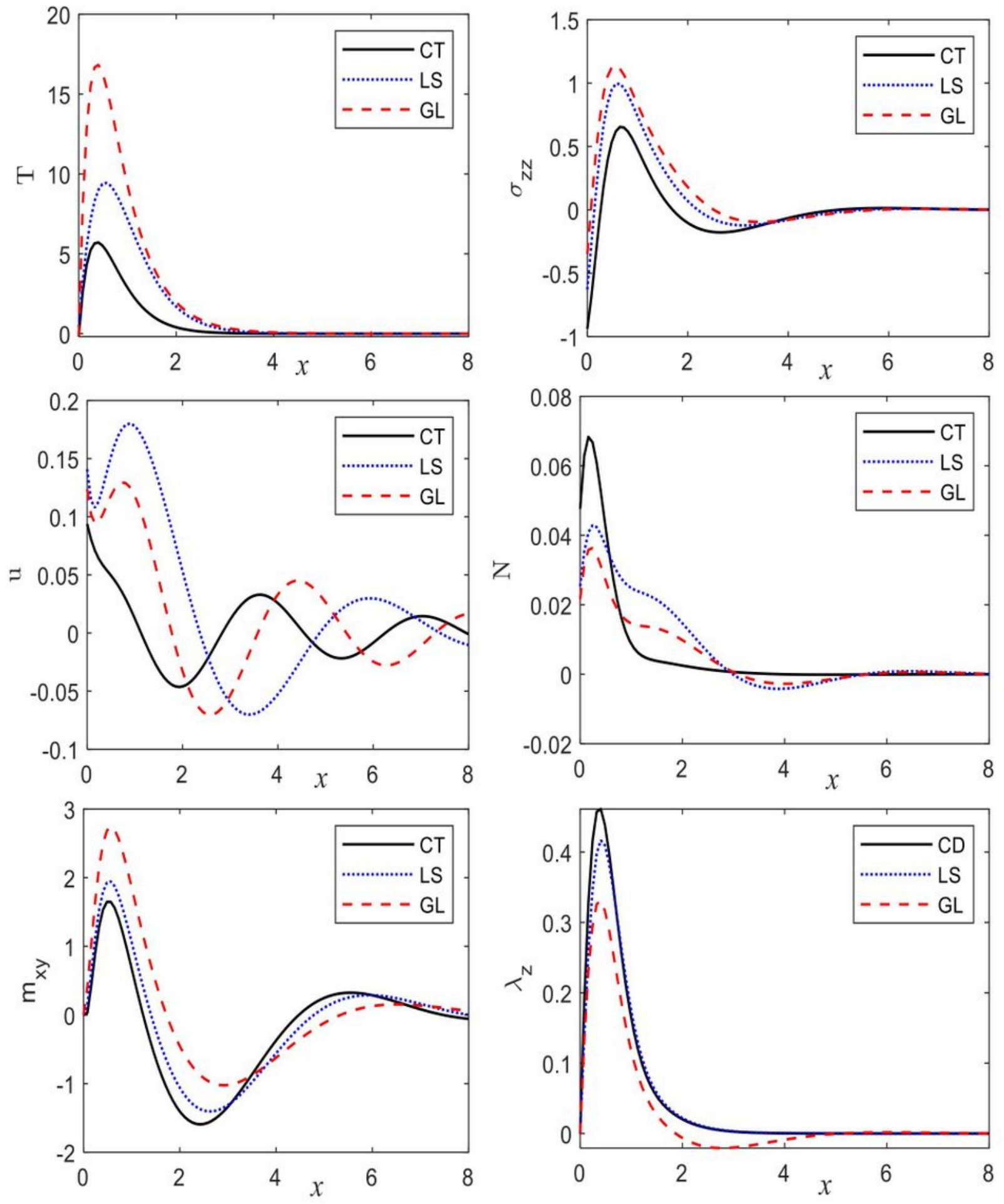

Figure 1

The variation of the main fields against the horizontal distance of Si medium under the influence of three different theories according to the thermal relaxation times. 

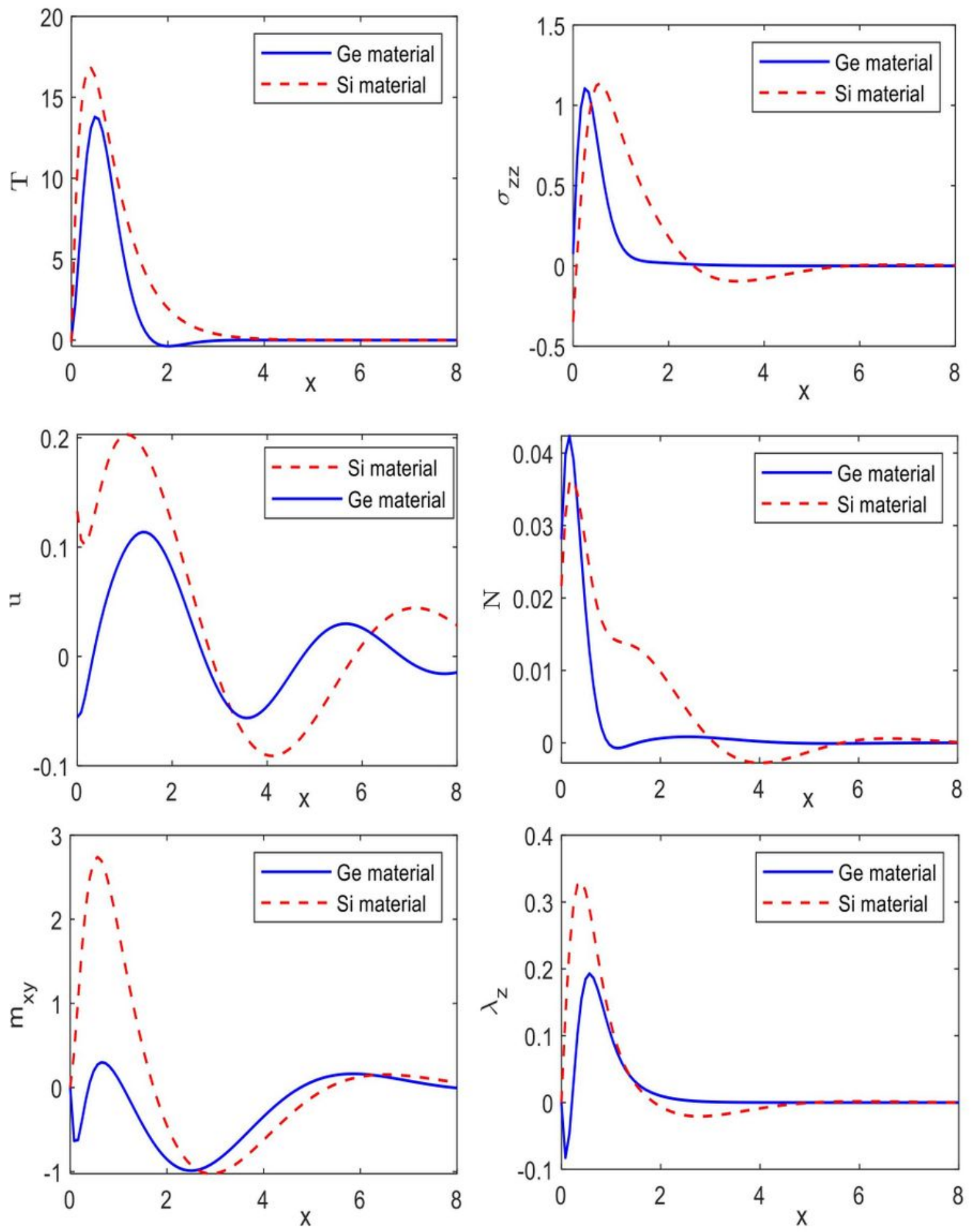

Figure 2

The comparison between Si and Ge materials of the main physical fields against distance in the generalized GL theory. 

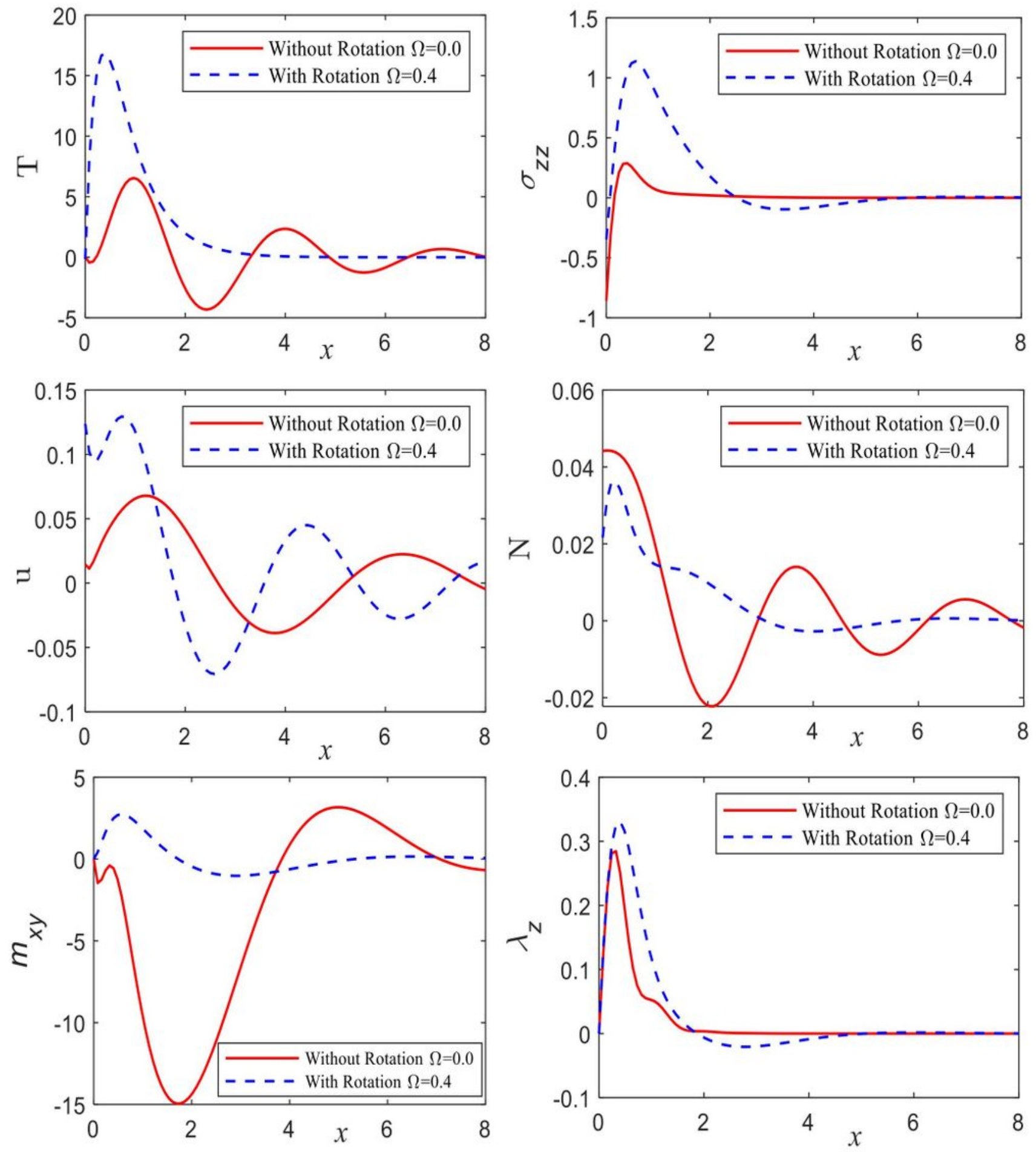

\section{Figure 3}

The comparison between the main physical fields against the horizontal distance under the effect of rotation parameters in the generalized GL theory of Si medium. 

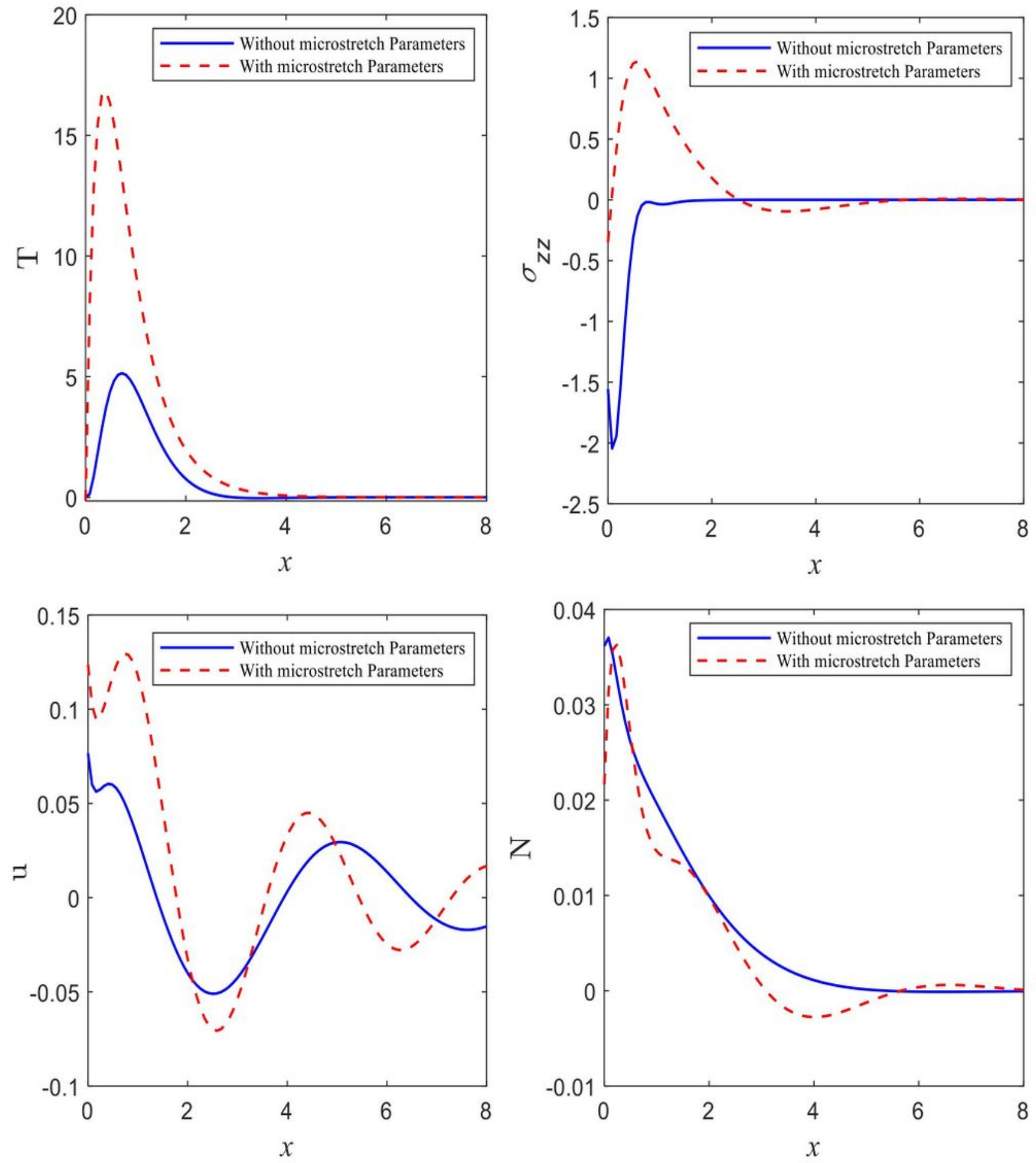

\section{Figure 4}

The comparison between the main physical fields against the horizontal distance under the effect of microstretch parameters in the generalized GL theory of Si medium.

\section{Supplementary Files}


This is a list of supplementary files associated with this preprint. Click to download.

- Geometryoftheproblem.png 